ARTICLE

\title{
Stream-specific feedback inputs to the primate primary visual cortex
}

\author{
Frederick Federer (10) ${ }^{1,4}$, Seminare Ta'afua ${ }^{1,2,4}$, Sam Merlin ${ }^{1,3}$, Mahlega S. Hassanpour ${ }^{1} \&$ \\ Alessandra Angelucci i(i) ${ }^{1 \times}$
}

The sensory neocortex consists of hierarchically-organized areas reciprocally connected via feedforward and feedback circuits. Feedforward connections shape the receptive field properties of neurons in higher areas within parallel streams specialized in processing specific stimulus attributes. Feedback connections have been implicated in top-down modulations, such as attention, prediction and sensory context. However, their computational role remains unknown, partly because we lack knowledge about rules of feedback connectivity to constrain models of feedback function. For example, it is unknown whether feedback connections maintain stream-specific segregation, or integrate information across parallel streams. Using viral-mediated labeling of feedback connections arising from specific cytochrome-oxidase stripes of macaque visual area $\mathrm{V} 2$, here we show that feedback to the primary visual cortex (V1) is organized into parallel streams resembling the reciprocal feedforward pathways. This suggests that functionally-specialized V2 feedback channels modulate V1 responses to specific stimulus attributes, an organizational principle potentially extending to feedback pathways in other sensory systems.

\footnotetext{
${ }^{1}$ Department of Ophthalmology and Visual Science Moran Eye Institute, University of Utah, 65 Mario Capecchi Drive, Salt Lake City, UT 84132, USA.

${ }^{2}$ Department of Biomedical Engineering, University of Utah, Salt Lake City, UT 84132, USA. ${ }^{3}$ Present address: Medical Science, School of Science, Western Sydney University, Campbelltown, Sydney, NSW 2560, Australia. ${ }^{4}$ These authors contributed equally: Frederick Federer, Seminare Ta'afua.

$\varpi_{\text {email: }}$ alessandra.angelucci@hsc.utah.edu
} 
$\mathrm{n}$ the primate sensory neocortex, information travels along multiple parallel feedforward (FF) pathways through a hierarchy of areas ${ }^{1}$. Neuronal receptive fields (RFs) in higherorder areas become tuned to increasingly complex stimulus features, with multiple parallel pathways specialized in processing specific attributes of sensory stimuli (e.g., object form versus motion). The role of FF connections in shaping cortical RFs has long been recognized ${ }^{2}$. In contrast, little is known about the function of feedback (FB) connections, although they have been implicated in top-down modulations of neuronal responses, such as attention ${ }^{3,4}$, prediction ${ }^{5,6}$, and sensory context ${ }^{7-10}$. One limitation to understanding the computational function of FB is that we lack information on the rules of FB connectivity to constrain models of FB function. For example, it is debated whether FB connections are anatomically diffuse and, therefore, unspecific with respect to the functional domains they contact in lowerorder areas, or whether they are patterned and functionally specific $^{11}$. A related question is whether diffuse and unspecific FB connections integrate information across parallel processing streams, or whether these connections maintain stream specificity, like their reciprocal FF pathways. Answering these questions would further our understanding of FB function and allow us to refine and inspire theories of cortical computation, many of which make specific assumptions about the functional specificity, or lack thereof, of FB connections ${ }^{12-16}$.

FB connections from the secondary visual area (V2) to the primary visual cortex (V1) in macaque monkey are well suited to address questions of anatomical and functional specificity and parallel FB pathways. This is because V2 is partitioned into cytochrome-oxidase (CO) stripe compartments that receive segregated FF projections from specific V1 CO compartments and layers ${ }^{17,18}$, and $\mathrm{CO}$ compartments in V1 and V2 have specialized functional properties and maps ${ }^{19,20}$. It is well established that CO blobs project predominantly to $\mathrm{V} 2 \mathrm{CO}$ thin stripes, while V1 interblobs project to V2 thick and pale stripes ${ }^{21,22}$. These projections arise predominantly from V1 layers (L) $2 / 3$ and $4 \mathrm{~B}$, and sparsely from $4 \mathrm{~A}$ and $5 / 6$, with $\mathrm{L} 4 \mathrm{~B}$ projecting more heavily to thick stripes compared to other stripe types ${ }^{23,24}$. However, it is debated whether V2-to-V1 FB connections form similar parallel pathways that segregate within V1 CO compartments ${ }^{25-27}$, therefore maintaining stream specificity, or diffusely project to all compartments ${ }^{28}$, thus integrating information across streams. This controversy is primarily due to the lack, in previous anatomical studies, of sensitive anterograde neuroanatomical tracers capable of labeling V2-to-V1 axons fully and selectively, without also labeling the reciprocal V1-to-V2 FF projections. In this work, using selective viral-mediated labeling of $\mathrm{FB}$ connections arising from specific V2 CO stripes, we show that, like V1-to-V2 FF pathways, V2 FB connections to V1 are organized into multiple parallel streams that segregate within the $\mathrm{CO}$ compartments of V1 and V2. These results suggest functionally specialized FB channels.

\section{Results}

To selectively label FB connections to $\mathrm{V} 1$ arising from specific V2 $\mathrm{CO}$ stripes, in 3 animals (7 injections), we first identified the V2 stripes in vivo, using intrinsic signal optical imaging. We then targeted to a particular V2 stripe type injections of a mixture of Cre-expressing and Cre-dependent adeno-associated virus, serotype 9 (AAV9), carrying the gene for green fluorescent protein (GFP) or tdTomato (tdT) (see "Methods"). In 2 animals (6 injections), injections were made blindly with respect to V2 stripe identity, and the stripe location of the injection sites determined postmortem. We have previously shown that in the primate visual cortex, this viral vector combination results in selective anterograde infection of neurons at the injected V2 site and virtually no retrograde infection of neurons in $\mathrm{V}^{8}$. We quantified the resulting distribution of labeled FB axons across V1 layers and $\mathrm{CO}$ compartments. We present results from a total of 13 viral injections made in area V2 of 5 macaque monkeys, spanning all V2 layers. First, we describe the distribution of V2 FB axon terminals across V1 layers ( $n=4$ injection cases), and subsequently their distribution relative to CO blobs and interblobs ( $n=9$ injection cases).

Laminar distribution of V2 feedback projections to V1. Here we present results from 4 injections of the anterograde viral mixture that were made blindly into V2. Alignment of the injection sites to V2 sections stained for CO (see "Methods") revealed that two AAV9-tdT injections were confined to thin stripes (one example is shown in Fig. 1a, b) and two AAV9-GFP injections encompassed all 3 stripe types, but were mostly confined to pale-lateral stripes (one example is shown in Fig. 1a, c). Following Federer et al. ${ }^{23}$, here we term pale stripes located lateral or medial to an adjacent thick stripe as pale-lateral or palemedial, respectively. Analysis of the laminar distribution of labeled FB axons resulting from these injections was performed on V1 tissue sectioned perpendicular to the layers along an axis parallel to the V1/V2 border. Fluorescent label was first imaged in sample sections, the same sections were then stained for $\mathrm{CO}$, to reveal layers, and finally the sections were re-imaged simultaneously for both $\mathrm{CO}$ and fluorescent signals, to maintain perfect alignment of the FB-label and CO-defined cortical layers (see "Methods"). Qualitative observations of sections imaged for GFP and tdT fluorescence (Fig. 1d and left panels of Fig. 1e, f), and quantitative analysis of fluorescent signal intensity across layers (right panels in Fig. 1e, f; see "Methods" for analysis details) demonstrated that V2 FB neurons terminate most densely in L1 and the upper part of L2, and in L5/6 (particularly 5B and 6). There were also weaker FB projections to the lower part of L2 and upper part of L3. A sparser projection to L4B, instead, was seen only after injections that encompassed thick and pale stripes, but not after injections confined to thin stripes. Little or no projections were seen in L4A and 4C. In the layers devoid of terminal FB axons, nevertheless, the axon trunks of labeled FB axons could be seen ascending vertically to the upper layers, but these axons did not send lateral branches in these layers.

Layer-by-layer statistical comparisons across stripe groups (thin vs. all stripes) revealed a significant difference in the projections to $\mathrm{L} 4 \mathrm{~B}$, which were virtually absent after thin stripe injections, but present when the injection encompassed also the pale/thick stripes. Moreover, injections in thin stripes resulted in significantly less projections to L6, compared to injections that additionally encompassed thick/pale stripes $(p<0.001$ for both L4B and L6 comparisons, Mann-Whitney U test; for L4B, $n=22$ independent samples from 2 independent thin stripe injection cases and $n=21$ independent samples from 2 independent thick/ pale-stripe injection cases; for L6: $n=19$ independent samples from 2 thin stripe injection cases, and $n=22$ for 2 thick/palestripe cases; samples consisted of bins of fluorescent intensity measurements as described in the "Methods"). Within each stripe group, we also statistically compared the resulting FB-label intensity across the sublayers of L4 $(4 \mathrm{~A}, 4 \mathrm{~B}, 4 \mathrm{C})$. We found no significant difference in the amount of $\mathrm{FB}$ projections from thin stripes to the different L4 sublayers (Fig. 1e). In contrast, following injections that additionally involved pale/thick stripes, L4B ( $n=21$ bins over 2 injection cases) showed significantly denser FB terminations compared to L4A ( $n=9$ bins over 2 injection cases) and $4 \mathrm{C}$ ( $n=40$ bins over 2 injection cases; $p<$ 0.001 for the L4B vs. L4A comparison, and $p<0.003$ for the L4B 

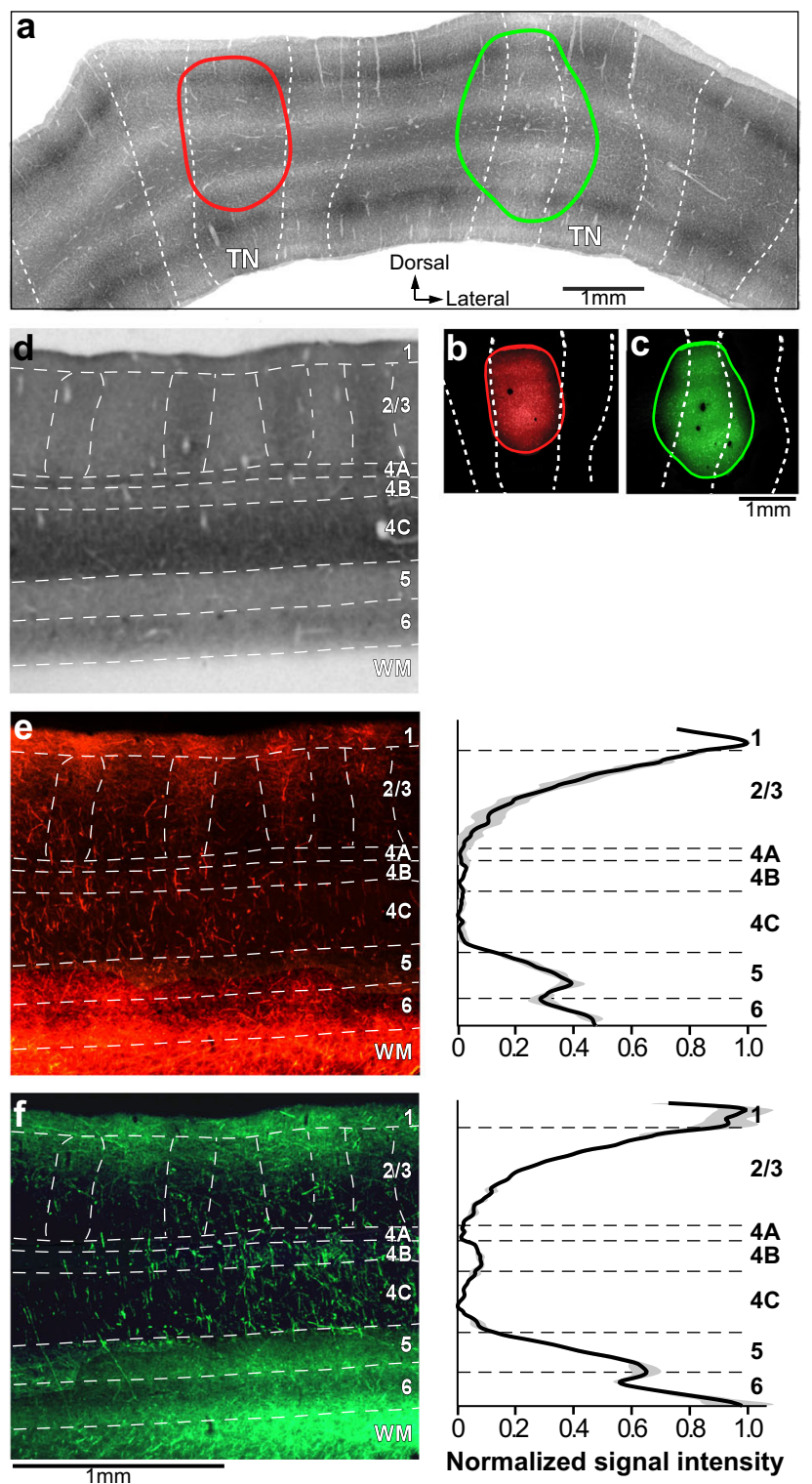

Normalized signal intensity

Fig. 1 Laminar distribution of V2 feedback projections to V1. Case MK359LH. a Micrograph of a merged stack of V2 sections stained for CO, to reveal the stripes (delineated by dashed white contours). Red and green ovals are the composite outlines of an AAV9-tdT and AAV9-GFP injection site, respectively, taken from $(\mathbf{b}, \mathbf{c})$ and overlaid onto the $\mathrm{V} 2 \mathrm{CO}$ stack. TN: thin stripes. b, c Micrographs of representative AAV9-tdT and AAV9-GFP injection sites taken under fluorescent illumination. While the images are from a single tissue section, the red and green outlines mark the full extent of the injection sites through the layers, and were generated by aligning images of injection sites through the depth of the cortex. $\mathbf{d}$ Image of CO-stained section through the layers of V1 (indicated on the right side). White dashed contours delineate layer boundaries, and the $\mathrm{CO}$ blobs in L2/3. The same tissue section, but imaged for fluorescent label, is illustrated in (e) and (f). e Left: Image of tdTlabeled FB axons through V1 layers resulted from the thin stripe injection shown in panels (a, b). Right: Population mean (solid line) \pm s.e.m. (gray shading) of normalized fluorescent signal intensity across $\mathrm{V} 1$ layers following thin stripe injections $(n=2)$. $\mathbf{f}$ Same as (e) but for FB label resulted from injections $(n=2)$ that were centered on a pale-lateral stripe, but spilled into adjacent thick and thin stripes. Statistical analysis of differences between stripe types is reported in the "Results". Scale bar under left panel in (f) is valid for (d) and left panels in $(\mathbf{e}, \mathbf{f})$. Results in (a-d), and left panels in $(\mathbf{e}, \mathbf{f})$ are representative of four independent injections centered in thin $(n=2)$ or pale $(n=2)$ V2 stripes. Source data for right panels in $(\mathbf{e}, \mathbf{f})$ are provided as a Source data file. vs. L4C comparison; Kruskal-Wallis test with Bonferroni correction), while L4A did not differ significantly from L4C. In summary, $\mathrm{L} 4 \mathrm{~B}$ receives significant $\mathrm{FB}$ projections from $\mathrm{V} 2$ thick/ pale stripes, but not from V2 thin stripes, and the latter project less heavily to L6 compared to thick/pale stripes.

Segregated V2-to-V1 feedback pathways. We next asked how FB projections from different V2 stripe types are distributed across the tangential plane of V1. Figure $2 \mathrm{a}-\mathrm{c}$ shows one example case in which, using intrinsic signal optical imaging to reveal functional V2 stripes in vivo (see "Methods", Optical Imaging), an injection of AAV9-GFP was targeted to a thin V2 stripe, and an injection of AAV9-tdT to the adjacent pale-medial stripe. Figure 2a shows the orientation difference map in two overlapping regions of interest (ROI1 and ROI2), and the spatial frequency (SF) difference map (low SF-high SF) for ROI2. In the orientation difference map, thin stripes can be identified as regions having weaker or no orientation responses, and pale stripes as regions with strong orientation responses neighboring a region with weak or no orientation domains. In the SF difference map, domains responsive to low SF are located in the thin and thick, but not pale stripes. The stripe location of the injection sites was confirmed postmortem on CO-stained V2 sections (Fig. 2b), which were aligned to the optical maps as described in the "Methods" and in Supplementary Fig. 1. Figure 2c demonstrates good correspondence of V2 stripes as defined in the orientation map, SF map, and $\mathrm{CO}$ staining. GFP- and tdT-labeled FB projections arising from each of these two injection sites formed terminal patches in the superficial layers of V1 (Fig. 2d). When the GFP and tdT images were merged, it was clear that, in the regions where both FB fields overlapped, GFP- and tdT-labeled patches were interleaved (Fig. 2d right panel). Importantly, only axon fibers and boutons, but not somata, were labeled in the FB projection zones, indicating these patches were, indeed, formed by axonal terminations of anterogradely-labeled FB neurons (Fig. 2e), but not retrogradely-labeled V1 cells. These results indicated that V2 FB projections arising from distinct stripe types segregate within V1.

We next asked how the terminal patches formed by $\mathrm{FB}$ projections arising from each of the stripe types relate to the $\mathrm{CO}$ blobs and interblobs of $\mathrm{V} 1$, and whether patchy terminations occur throughout the V1 layers of FB termination. Below we present results from a total of nine viral injections that were confined to V2 thick $(n=3)$, thin $(n=3)$, or pale $(n=3)$ stripes.

V2 thick stripes project to V1 interblobs. Here we present results from a total of three viral injections (2 AAV9-GFP, 1 AAV9-tdT, $n=3$ animals) that were confined to V2 thick stripes. Analysis of the distribution of labeled $\mathrm{FB}$ axons relative to the $\mathrm{CO}$ compartments of $\mathrm{V} 1$ was performed on tissue sectioned parallel to the pial surface. Fluorescent label was imaged in sample sections through the layers where FB axons terminated, namely L1A, $1 \mathrm{~B}, 2 / 3,4 \mathrm{~B}$, and $5 / 6$; the same or adjacent sections were then stained for $\mathrm{CO}$ to reveal layers and $\mathrm{CO}$ blobs, and imaged. Images of each section were vertically aligned in a sequential stack through the depth of V1, by aligning the radial blood vessels (as shown in Supplementary Fig. 1d). One example AAV9-GFP injection site confined to a thick stripe is shown in Fig. 3, and the FB axons in V1 labeled from this injection are shown in Fig. 4 and Supplementary Fig. 2. The left panel of Fig. 4a shows a filtered CO-stained section through V1 L2/3 overlapping the FB termination zone. The CO blob outlines extracted from this image (see "Methods") are shown superimposed to the GFP-labeled axons through the depth of V1 (left panels in Fig. 4b-f). Qualitative observation of these images revealed patchy $\mathrm{FB}$ terminations in $\mathrm{V} 1$, with the densest patches located preferentially in the 
a $\mathrm{OI}$

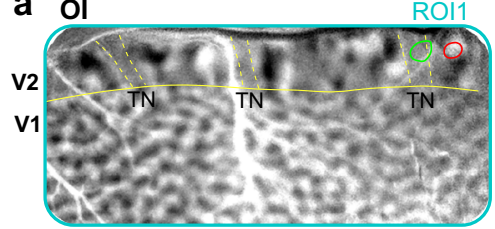

b co

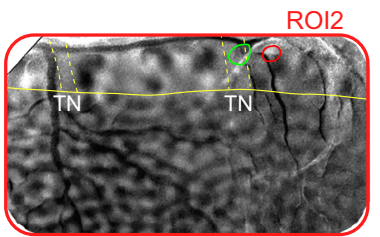

$\overline{2 m m}$

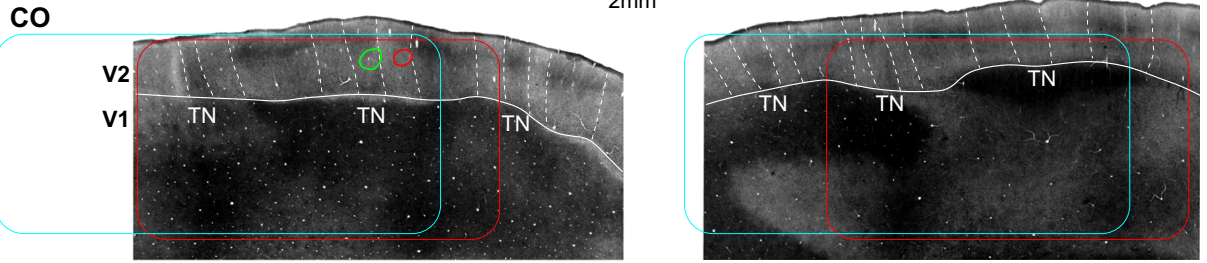

C V2 injections
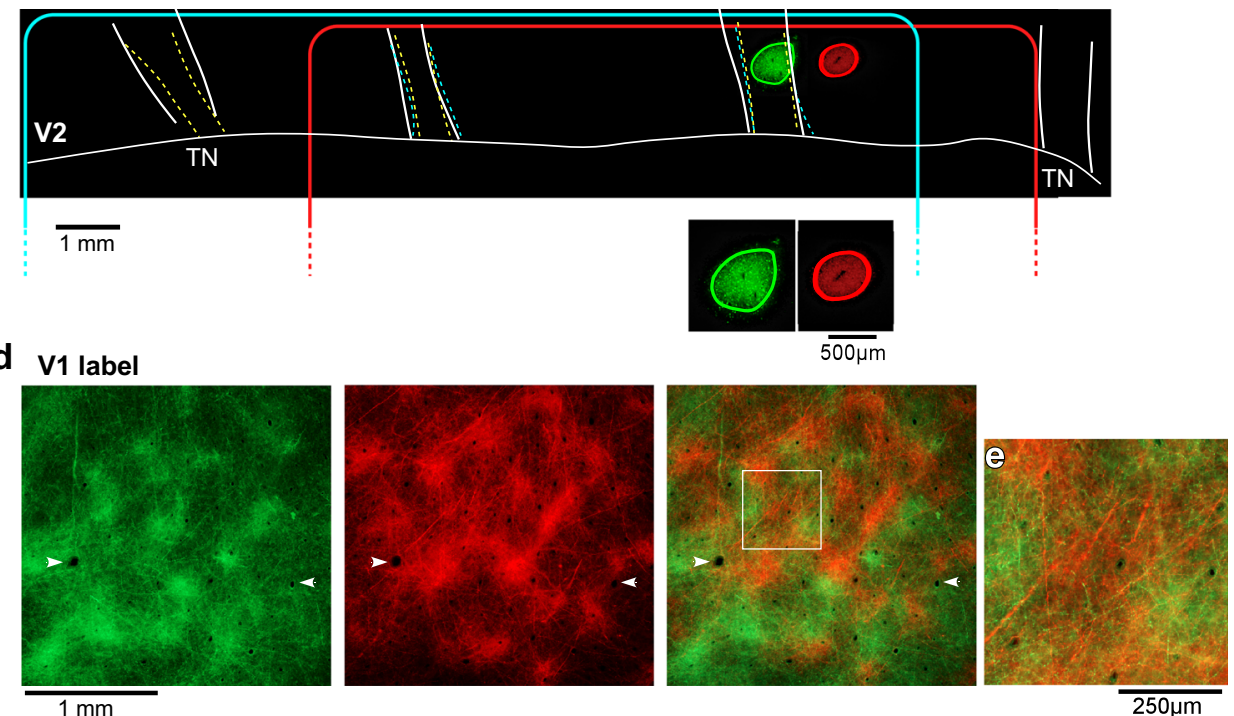

Fig. 2 Segregated V2-to-V1 feedback pathways. Case MK374RHLat. a Functional maps recorded using optical imaging (OI). Left and middle: Orientation difference map in two overlapping regions of interest (ROI1 and 2) encompassing $\mathrm{V} 1$ and $\mathrm{V} 2$, obtained by subtracting responses to achromatic gratings of two orthogonal orientations (ROI1: 45-135'; ROI2: 90- ${ }^{\circ}$ ). Solid yellow contour: V1/V2 border based on the orientation map. Dashed yellow contours delineate the thin stripes (TN; regions of weak orientation responses). Right: SF difference map in ROI2, obtained by subtracting high-SF from low-SF responses. Horizontal solid cyan contour: V1/V2 border based on the orientation map. Vertical cyan contours delineate TN (dashed) and thick (solid) stripes, as regions having low-SF responsive domains (darker regions). b CO-stained tangential tissue sections $240 \mu \mathrm{m}$ apart (left one is more superficial), encompassing $\mathrm{V} 1$ and $\mathrm{V} 2$, revealing the stripes and the location of the two injection sites (ovals); the latter were determined by alignment of fluorescent label to CO-stained sections (see Supplementary Fig. 1). Solid white contour: V1/V2 border based on CO staining. Dashed white contours delineate the V2 stripes. Cyan and red box: locations of optically imaged ROI1 and 2, respectively, on the CO-stained sections. Scale bar in (a) valid for all panels in (a, b). c Fluorescent micrographs of the AAV9-GFP and AAV9-tdT injection sites (shown at higher power in the inset below), outlined in green and red, respectively, with superimposed outlines of TN stripes based on CO (white) and OI maps (yellow, orientation, cyan, SF). Note the good correspondence of $\mathrm{CO}$ - and functionally-defined stripes. The injection outlines are shown overlaid to the V2 optical maps in (a) and CO sections in (b). d Micrograph of a V1 section through L1B of V1 seen under GFP (LEFT) and tdT (MIDDLE) fluorescence, and with the two channels merged (RIGHT). Arrowheads point to same blood vessels in all panels. GFP and tdT-labeled patches are interleaved. Label inside the white box is shown at higher power in (e), to demonstrate lack of labeled somata, indicating anterograde viral expression. Results in (a-e) are representative of six independent injections centered in thin ( $n=3$ ) or pale $(n=3)$ V2 stripes.

interblobs, across all layers of FB termination. Consistent with the laminar analysis shown above, the densest terminations were located in L1/upper 2 and 5B-6, and sparser projections in $\mathrm{L} 2 / 3$ and $4 \mathrm{~B}$. To quantify the distribution of $\mathrm{FB}$ projections in the blobs and interblobs of $\mathrm{V} 1$, for each layer we measured fluorescent signal intensity within a square region of interest (ROI) centered on each blob overlaying the FB termination zone, and encompassing the average blob and interblob diameter in the $\mathrm{CO}$ map (see "Methods" and Supplementary Fig. 3a, c). Fluorescent signal intensity was, then, summed across all blob ROIs within a layer, and normalized to the maximum intensity value, to generate a heatmap of fluorescent signals for that layer (Supplementary Fig. 3d, Fig. 4b-f middle panels). The black circle on each layer heatmap indicates the average blob diameter estimated from the blobs used to compute the heatmap for that specific layer (as described in the "Methods" and in Supplementary Fig. 3a, b). In all layers, the highest fluorescence intensity lay outside the average blob border, indicating the densest FB termination lay in the interblobs. The densest FB label, in this case, was located in the upper right quadrant of the heatmaps, rather than uniformly filling the entire interblob region all around the blob. Indeed the densest FB label was consistently located to one 


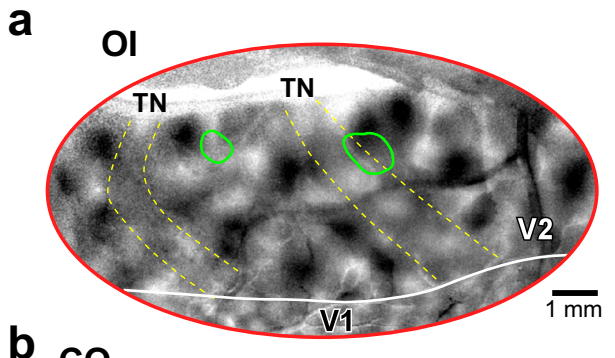

b co
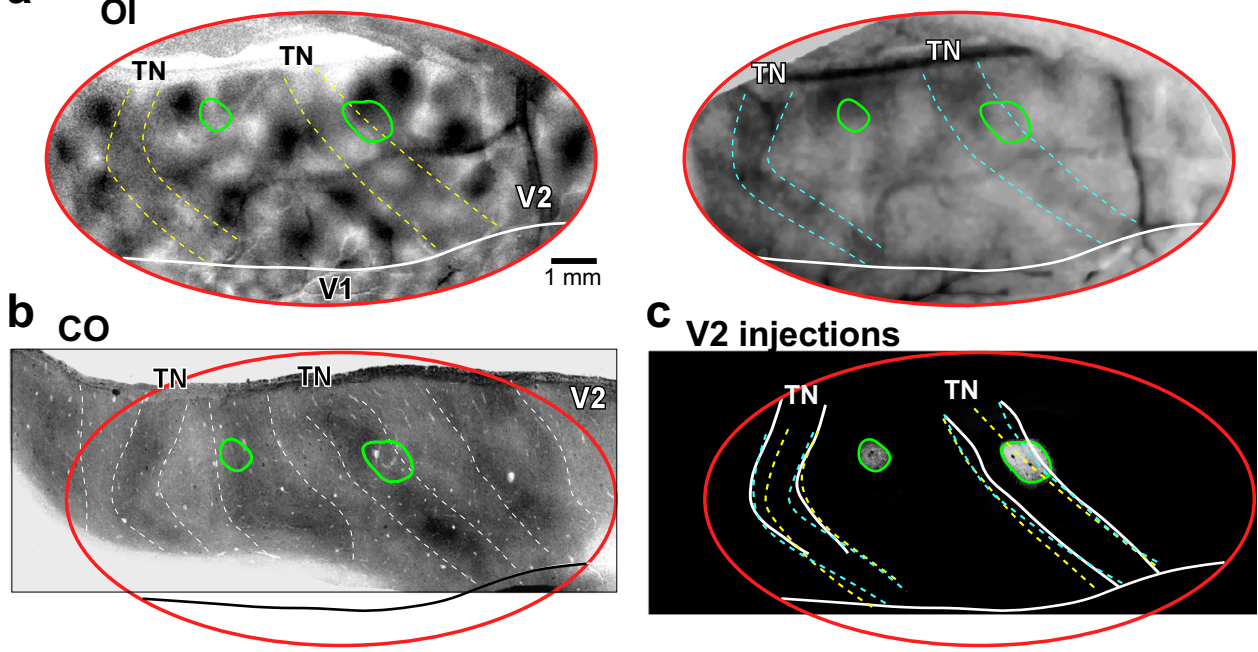

Fig. 3 Injection sites in V2 thick and thin stripes. Case MK356RH. Two AAV9-GFP injection sites, one confined to a V2 thick stripe, the other to a thin stripe. a Orientation (45-135\%; left) and SF (low-high; right) difference maps in V2. Dashed contours delineate regions having weak orientation responses (yellow), and preferring low SFs (cyan), corresponding to thin stripes; solid white contour: V1/V2 border based on the orientation map.b Stack of aligned and merged tangential V2 tissue sections stained for CO, revealing the stripe pattern. Red oval: imaged region from (a), determined based on the same alignment procedure described for a different case in Supplementary Fig. 1. Dashed white contours outline the TN and thick stripes; solid black contour: V1/ V2 border based on the orientation map. c Micrographs of the two AAV9-GFP injection sites taken under fluorescent illumination and outlined, with superimposed outlines of TN stripes based on CO (white) and OI maps (yellow, orientation, cyan, SF). Note the good correspondence between functionally-defined and CO-defined stripes. The outlines of the injection sites (green ovals) indicate the location of the injection sites on CO sections (b) and OI (a) maps. These injection sites in ( $\mathbf{a}-\mathbf{c})$ are representative of six independent injections centered in thin $(n=3)$ or thick $(n=3)$ 2 stripes.

side of the blobs, in the termination zone, perhaps due to the small injection site being confined to few orientation domains in the functional map of V2 (Fig. 3a), or due to the topographical organization of FB connections, which precisely interconnect matching retinotopic sites within the V1 and V2 CO compartments. The heatmap for L4B showed much lower fluorescent label intensity compared to the other layers, but branching axons were clearly visible in this layer (Fig. 4e, left panel), and the highest label intensity was still found within the interblobs (Fig. 4e, right panel).

From each layer heatmaps, we generated graphs of the normalized fluorescence signal intensity as function of distance from the center of the average blob (blue curves in right panels of Fig. 4b-f), as described in the "Methods" and Supplementary Fig. 3e. These curves were compared to similarly computed curves of CO signal intensity as function of distance from the center of the average blob (black curves in right panels of Fig. $4 \mathrm{a}-\mathrm{f}$, which were computed from the $\mathrm{CO}$ heatmap shown in the middle panel of Fig. $4 \mathrm{a}$, as described in the "Methods" and in Supplementary Fig. 3a, b, e). In all layers, the CO and fluorescent signal curves showed similar trends, peaking at the largest distance from the blob center, indicating that the highest fluorescent signals coincided with the brightest regions in $\mathrm{CO}$ staining, i.e., the interblobs.

The other two thick stripe injection cases showed similar results to the case shown in Fig. 4, albeit, due to the smaller size of these injections, the resulting V1 FB label was overall less dense and showed even greater specificity for the interblobs. Figure $7 \mathrm{a}$ summarizes the thick stripe population data for all layers analyzed.

V2 thin stripes project to V1 blobs. Three viral injections (2 AAV9-GFP, 1 AAV9-tdT, $n=3$ animals) were confined to V2 thin stripes. One example AAV9-GFP injection site and resulting
FB label in V1 are shown in Fig. 2. A second example AAV9-GFP injection site largely confined to a thin stripe (but slightly encroaching into the adjacent pale-lateral stripe) is shown in Fig. 3, and the FB axons in V1 labeled from this injection site are shown in Fig. 5 and Supplementary Fig. 2. Analysis of the distribution of FB labeled axons was performed as for the thick stripe injection case described above. Qualitative observation demonstrated patchy FB terminations, densest in V1 L1/upper 2 and $5 \mathrm{~B}-6$, and sparser in L2/3, with little or no projections to L4B (Fig. 5b-f, left panels). Both qualitative and quantitative analyses revealed that the densest patches of labeled FB axons lay preferentially inside the blobs, although some sparser label also occurred in the interblobs, likely due to the injection site encroaching into the adjacent pale stripe (Fig. 5b-f, middle and right panels). The heatmap for $\mathrm{L} 4 \mathrm{~B}$ showed no significant label (Fig. 5e, middle panel), with noisy background label in this layer being equally distributed across blobs and interblobs (Fig. 5e, right panel). Qualitative observations of L4B revealed only punctate GFP label with little or no lateral axon branching, suggesting this layer mainly contained unbranched axon trunks traveling vertically (i.e., orthogonal to the plane of imaging) toward the superficial layers. This was in contrast to the branched axonal label seen in this layer after both thick (Fig. 4e, left panel) and pale (Fig. 6e, left panel) stripe injections.

The other two thin stripe injection cases showed similar results to the case shown in Fig. 5, but since these injection sites were more clearly confined to thin stripes, the terminal FB label showed even greater specificity for blobs. Figure $7 \mathrm{~b}$ shows the thin stripe population data for all layers analyzed.

V2 pale stripes project to V1 interblobs. Three viral injections were confined to V2 pale stripes (1 AAV9-tdT in a pale-medial stripe and two AAV9-GFP in a pale-lateral stripe, $n=2$ animals). 

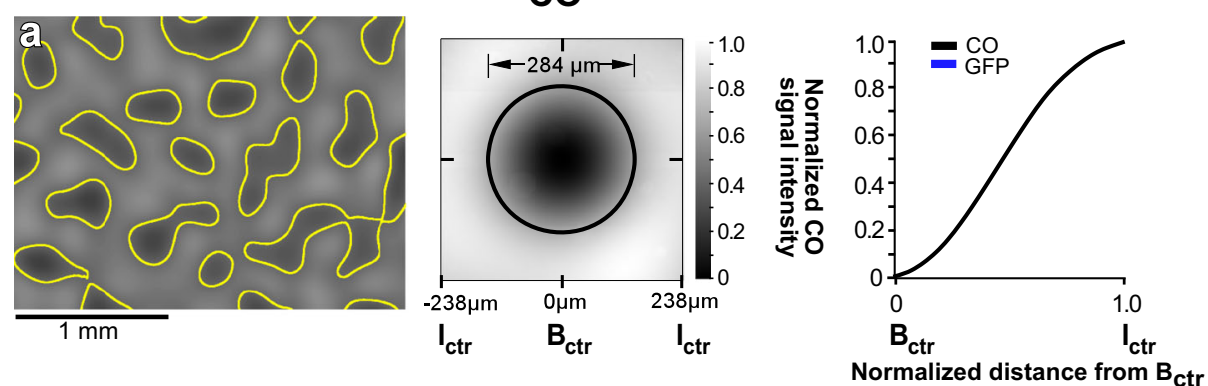

FB LABEL - Thick Stripes
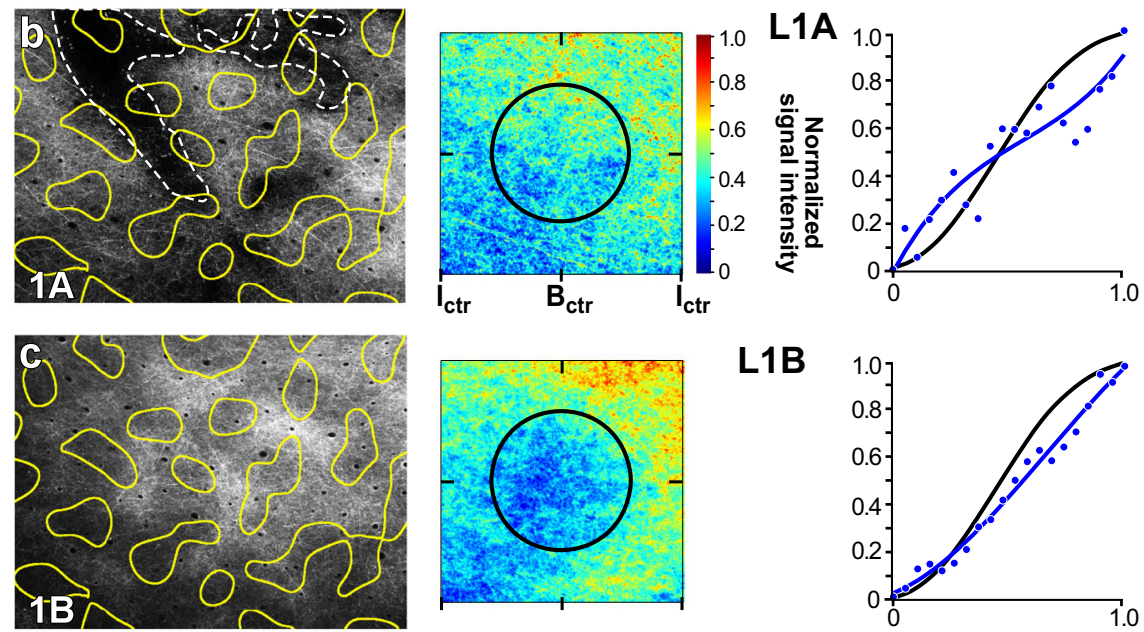

L1B
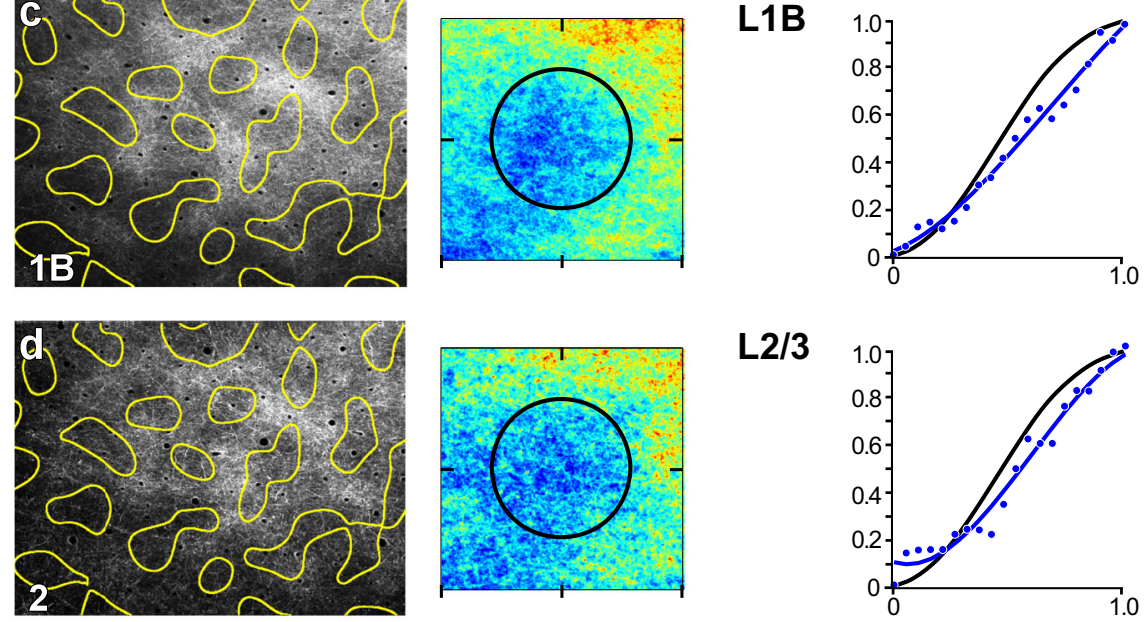

L2/3
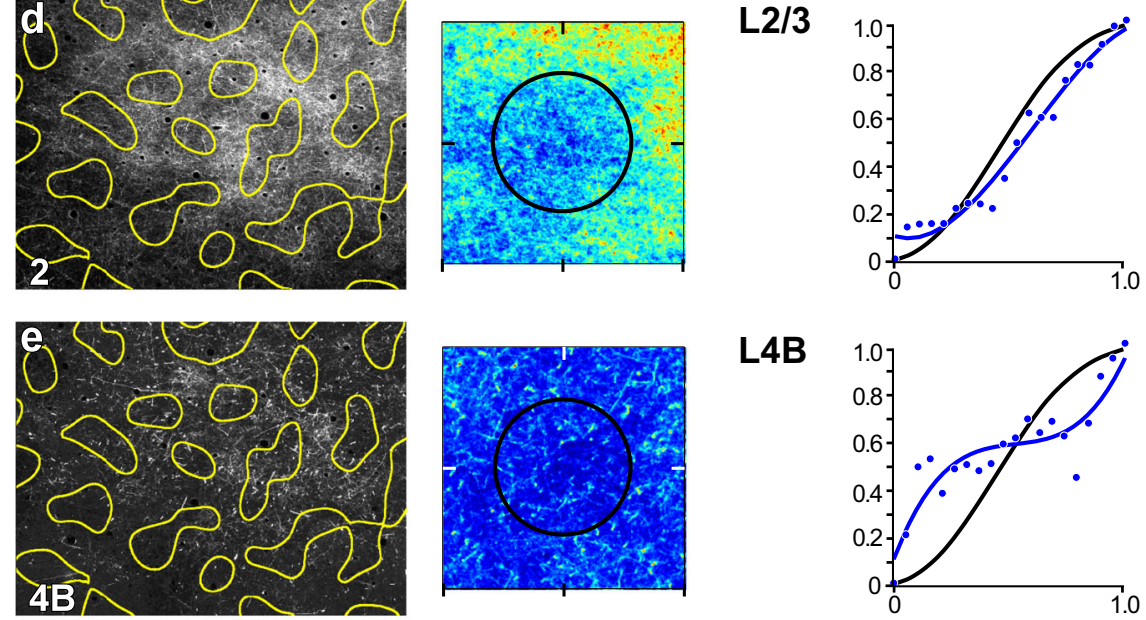

L4B
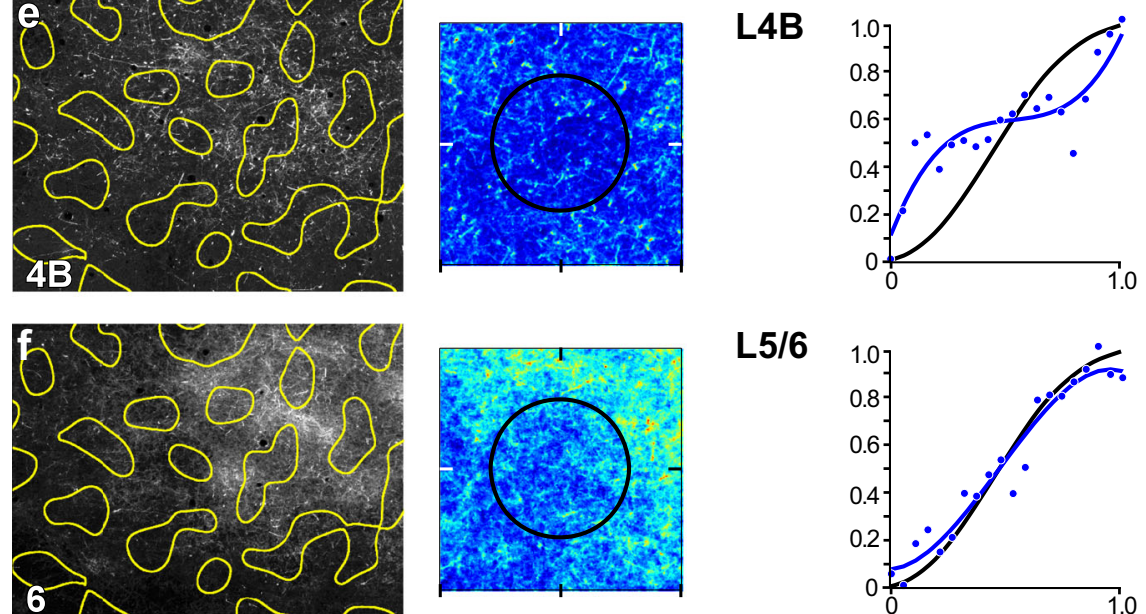

L5/6

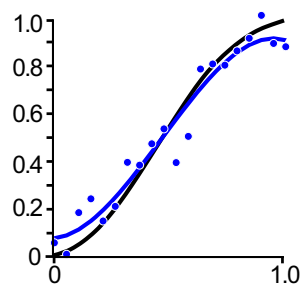

One example AAV9-tdT injection site confined to a pale-medial stripe is shown in Fig. $2 \mathrm{a}-\mathrm{c}$, and the FB axons in V1 labeled from this injection site are shown in Figs. 2b-e and 6. FB axons from this case also showed patchy FB terminations, densest in V1 L1/ upper 2 and 5B-6, and sparser in L2/3 and L4B (Fig. 6b-f, left panels). FB terminals in $\mathrm{L} 4 \mathrm{~B}$ formed clear lateral branches indicative of terminations within the layer. Both qualitative and quantitative analyses revealed that the densest patches of labeled FB axons in all layers lay preferentially in the interblobs (Fig. 6b-f, middle and right panels). Other pale-stripe injection cases showed similar results. Figure $7 \mathrm{c}$ summarizes the pale-stripe population data for all layers analyzed. 
Fig. 4 V2 thick stripes project to V1 interblobs. Case MK356RH. GFP-labeled FB axons in V1 after the thick stripe injection shown in Fig. 3. a Left: filtered CO map from a section through V1 L2. Segmenting out the darkest 33\% of pixels outlines the CO blobs (yellow). Scale bar valid also for (b-f, left). Middle: heatmap of CO signal intensity, generated by measuring CO intensity within a $476 \mu \mathrm{m}^{2} \mathrm{ROI}$ centered around each blob, then summing all ROIs and normalizing to maximum intensity (see "Methods" and Supplementary Fig. 3a, b). The average blob diameter (black circle) for this case was $284 \mu \mathrm{m}$. Right: normalized CO signal intensity as a function of distance from the blob center ( $B_{c t r}$ ), computed from the CO heatmap, as described in the "Methods" and Supplementary Fig. 3b, e. CO intensity is darkest in the $\mathrm{B}_{\mathrm{ctr}}$ and brightest in the interblob center $\left(\mathrm{I}_{\mathrm{ctr}}\right)$. The black CO-intensity curve is repeated on each plot in (b-f, right) for comparison with the FB-label intensity curves. (b-f) Left: GFP-labeled FB axons in different V1 layers (indicated at the bottom left corner) with superimposed the blob outlines (yellow) from (a, left). Dashed white lines in (b) outline the pial vessels. MIDDLE: heatmaps of the GFP-label intensity for each layer, generated by measuring fluorescent signal intensity within an ROI centered on each blob overlaying the FB label in that layer, summing all ROIs, and normalizing to maximum intensity. The size of the ROI in each layer is the average blob and interblob size within the FB termination zone in that layer (see "Methods" and Supplementary Fig. 3a, c). Black circles: average blob diameter for that layer. Right: plots of normalized CO (black curves) and GFP-fluorescence (blue curves) intensities as a function of distance from the $B_{c t r}$, computed as described in the "Methods" and Supplementary Fig. 3b, $d$, e. Here and in Figs. 5-7 right, the data were fitted with the following function: $y=a x^{3}+b x^{2}+c x+d$. In all layers, GFP-label intensity dominates in the interblobs. Results in (a-f) are representative of three independent injections centered in thick stripes. Source data for right panels in (a-f) are provided as a Source data file.

Population analysis. Figure 7 shows the summary data (see "Methods") for the population of injections, grouped by stripe type $(n=3$ injections per stripe type). Like the individual example cases, the population data shows that $\mathrm{FB}$ projections arising from $\mathrm{V} 2$ thick and pale stripes terminate preferentially in the interblob regions of $\mathrm{V} 1$, across all layers of $\mathrm{FB}$ termination (Fig. 7a, c, respectively). In contrast, $\mathrm{FB}$ projections arising from V2 thin stripes terminate preferentially in the CO blobs (Fig. 7b). Moreover, thick and pale, but not thin, stripes send FB projections to L4B. Statistical comparison of the CO-compartment location of densest FB label across stripe groups, for each V1 layer, indicated no significant difference between pale and thick stripes (L1A: $\mathrm{p}=0.33, n=20$ for pale, $n=17$ for thick; L1B: $p=$ 0.38, $n=28$ for pale, $n=15$ for thick; L2/3: $p=0.99, n=15$ for pale, $n=21$ for thick; L4B: $p=0.6, n=18$ for pale, $n=12$ for thick; L5/6: $p=0.98, n=26$ for pale, $n=18$ for thick; Kruskal-Wallis test with Bonferroni correction; $n$ represents the number of bins within which fluorescent intensity was measured, over three independent injection cases for all comparisons, as described in the "Methods"). In contrast, the CO-compartment location of FB projections to V1 differed significantly between thick and thin stripes, as well as between pale and thin stripes, and this was the case for all layers of FB termination $(p<0.001$ for all comparisons and all layers but L4B for which $p=0.008$ for the thick versus thin comparison), except for L4B for the thin versus pale-stripe comparison which did not reach statistical significance $(p=0.053)$, likely due to the low sample size (Kruskal-Wallis test with Bonferroni correction; the number of intensity bins examined over three independent injections for all thick vs. thin and pale vs. thin comparisons: L1A thick $=17$, thin $=27$, pale $=20$; $\mathrm{L} 1 \mathrm{~B}$ thick $=15$, thin $=19$, pale $=28 ; \mathrm{L} 2 / 3$ thick $=21$, thin $=20$, pale $=15 ; \mathrm{L} 4 \mathrm{~B}$ thick $=12$, thin $=11$, pale $=18 ; \mathrm{L} 5 / 6$ thick $=18$, thin $=20$, pale $=26$ ). Moreover, when all layers data were pooled together for each stripe group (see "Methods"), V2 thick and pale stripes showed no significant difference in the V1 COcompartment location of their projections $(p=0.801)$, while thick versus thin, and thin versus pale stripes were significantly different from each other $(p<0.001$ for all comparisons, Kruskal-Wallis test with Bonferroni correction, thick $n=83$ bins, thin $n=97$, pale $n=96$ ).

\section{Discussion}

We have shown that FB projections arising from the different $\mathrm{CO}$ stripes of V2 do not diffusely and unspecifically contact all V1 regions within their termination zones, but rather form patchy axon terminations which largely segregate in the CO compartments of V1: thin stripes project predominantly to blobs, and thick and pale stripes predominantly to interblobs. Moreover, all stripe types send densest projections to layers 1/upper 2 and 5B-6, and sparser projections to lower layer 2 and layer 3. However, only thick and pale, but not thin, stripes project to L4B (Fig. 8).

There has been a long-standing controversy regarding the clustering and specificity, or lack thereof, of FB connections to V1, mainly attributable to the technical limitations of the neuroanatomical tracers used in previous studies ${ }^{11}$. Conventional tracers used to label FB axons were either transported anterogradely, but had poor sensitivity and resolution (e.g., tritiated aminoacids, WGA-HRP, and first-generation adenoviral vectors), or had good sensitivity, but were transported bidirectionally (CTB, BDA), thus labeling the axons of both FF- and FBprojecting neurons. Studies using the first group of tracers injected in areas V2 or MT concluded that FB connections to V1 are diffuse, i.e., they make non-clustered terminations in their target areas, which are non-specific with respect to the $\mathrm{CO}$ compartments or functional domains of $\mathrm{V} 1^{28-30}$. Curiously, however, one such study ${ }^{28}$ showed at least one example of patchy FB axon terminations in layer 1 of macaque $\mathrm{V} 1$, but the authors did not comment on the modularity of FB connections. Studies using injections of bidirectional tracers into $\mathrm{V} 2^{25,27}$ or $\mathrm{V} 3^{11}$, instead, provided evidence for clustered $11,25,27$ and functionally specific $^{11,27} \mathrm{FB}$ projections to $\mathrm{V} 1$. Due to the bidirectional transport of the tracers used in the latter studies, however, it was unclear whether the axon clustering reflected the termination patterns of FB axons or of the axonal collaterals of the reciprocal FF projection neurons, known to form patchy connections ${ }^{21,22,31}$. For the same reason, it had remained unclear whether FB projections to V1 terminate only in L1 and 6 (which do not send, or only send very sparse FF projections to extrastriate areas), or also terminate in layers that send FF projections to higher cortical areas (such as L2/3 and $4 \mathrm{~B}$ ). In a few studies, FB axons were labeled by bulk injections of PHA-L or BDA in V2 or MT, and reconstructed through serial sections ${ }^{32-34}$. While these studies were not affected by ambiguity in the interpretation of the origin of axonal label, they, however, provided a limited sample of partially reconstructed FB axons.

To overcome the limitations of previous anatomical studies, in this study we have taken advantage of recent advances in neuroanatomical labeling methods based on the use of viral vectors to deliver genes for fluorescent proteins ${ }^{35}$. Specifically, we have used a mixture of AAV9-Cre and Cre-dependent-AAV9 to express fluorescent proteins in V2 FB neurons projecting to V1 without simultaneously labeling the axons of the reciprocal V1-to-V2 FF projection neurons. Our results resolve existing controversies by demonstrating unequivocally that FB connections from V2 to V1 form patchy terminations, in agreement with Angelucci et al. ${ }^{25}$ and Shmuel et $\mathrm{al}^{27}$, and that the $\mathrm{FB}$ terminal patches are 

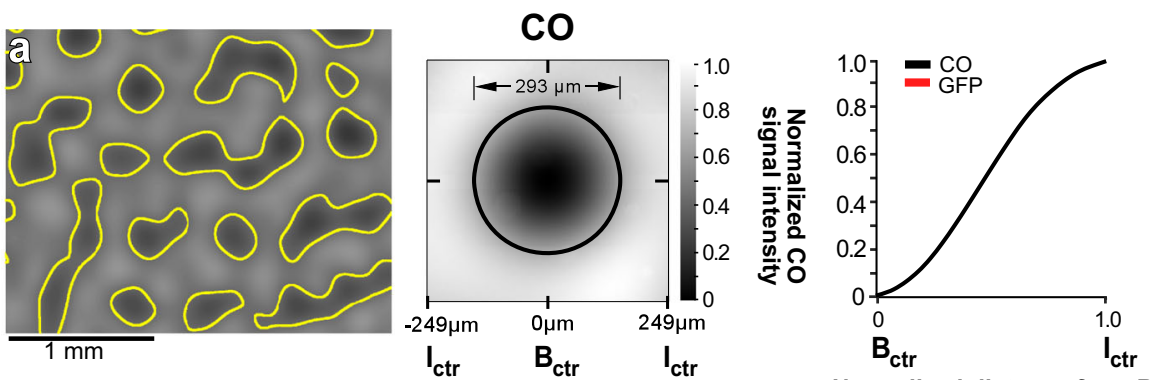

FB LABEL - Thin Stripes
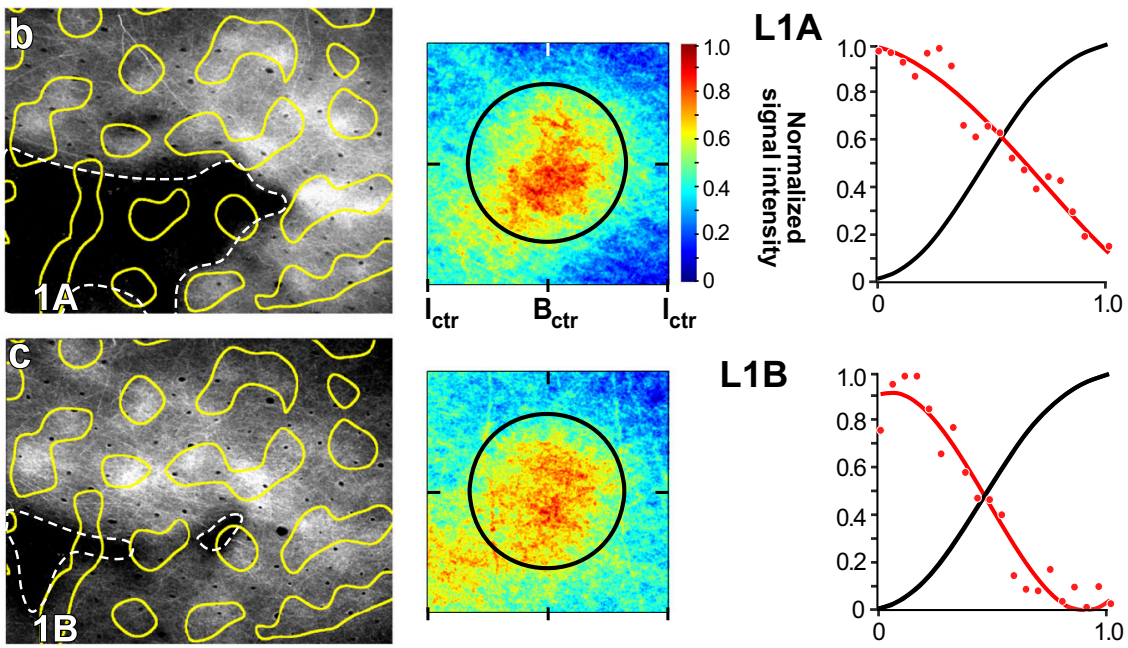

L1B
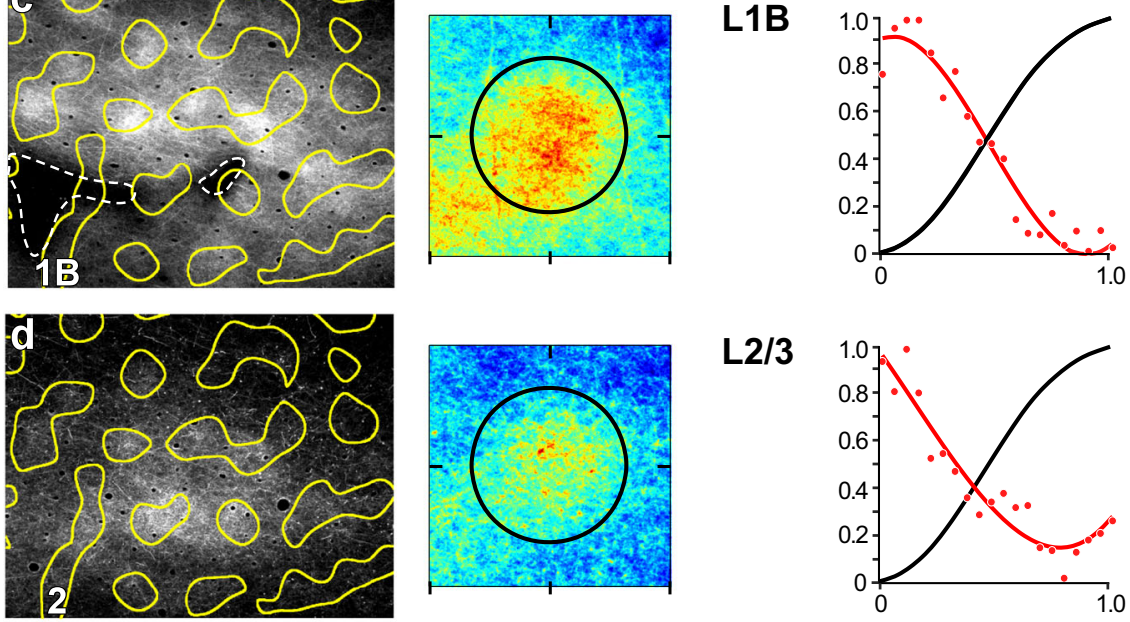

L2/3
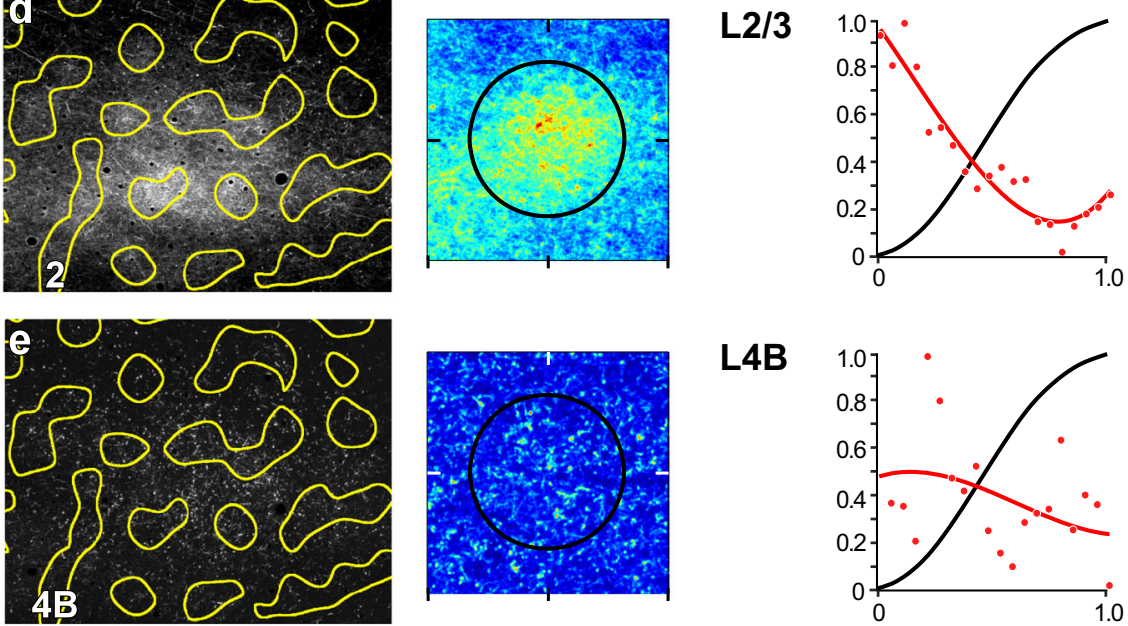

\section{L4B}
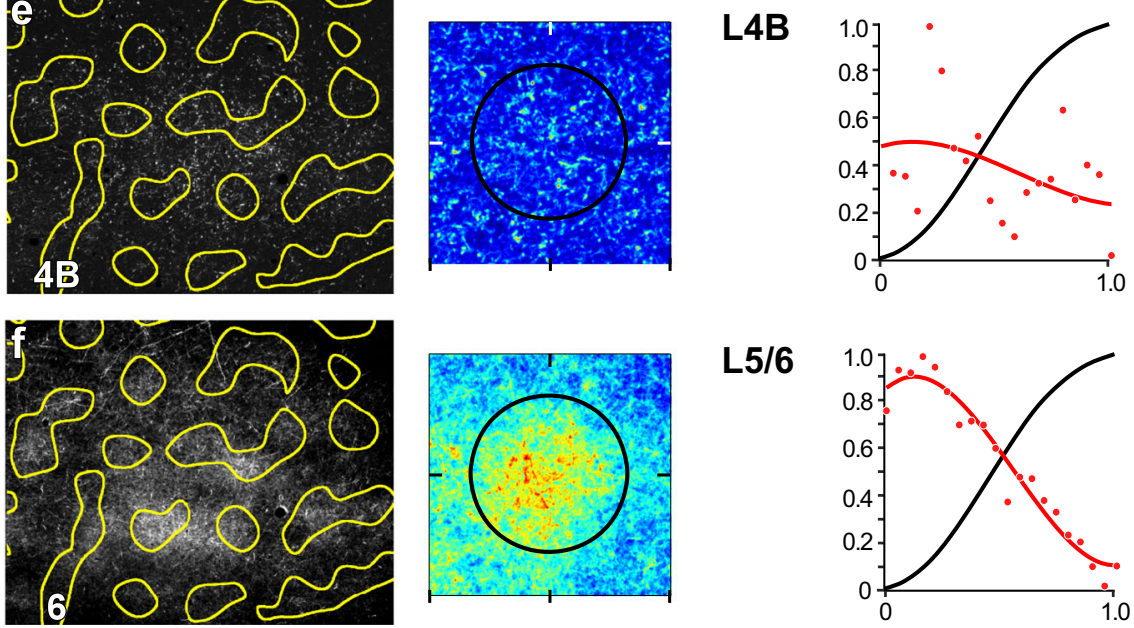

Fig. 5 V2 thin stripes project to V1 blobs. Case MK356RH. Same as in Fig. 4, but for GFP-labeled FB axons in V1 resulting from the AAV9-GFP injection site in a V2 thin stripe shown in Fig. 3. In all layers, GFP-label intensity dominates inside blobs. Results in (a-f) are representative of three independent injections centered in thin stripes. Source data for right panels in (a-f) are provided as a Source data file. 

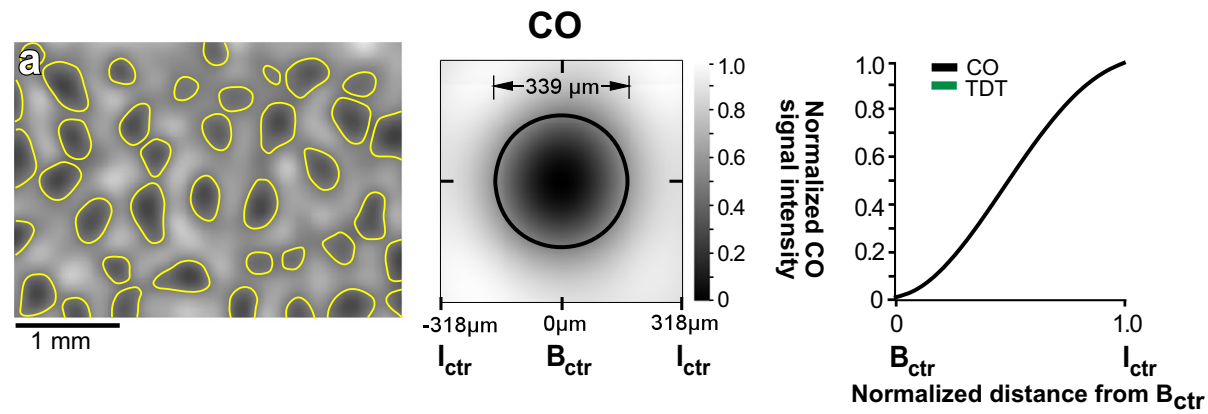

FB LABEL - Pale Stripes
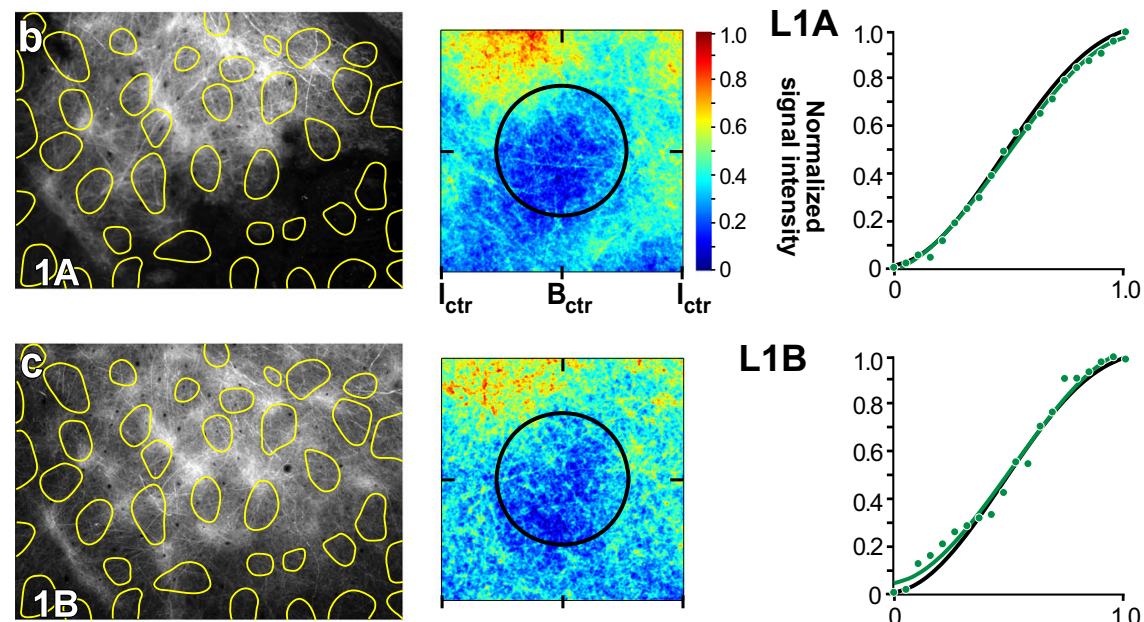

L1B
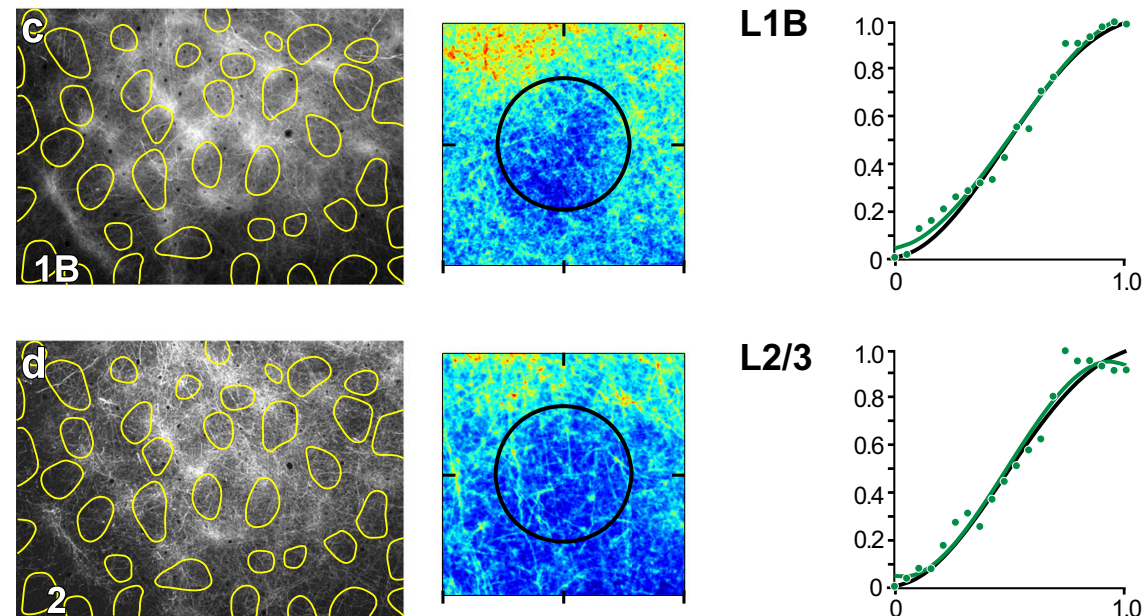

L2/3
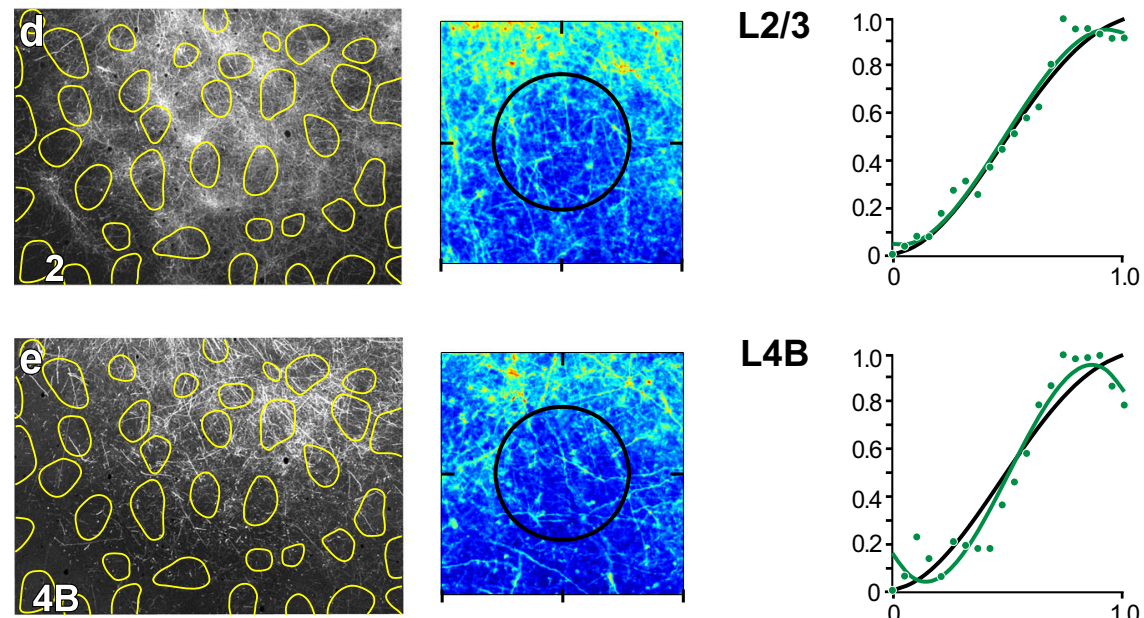

L4B
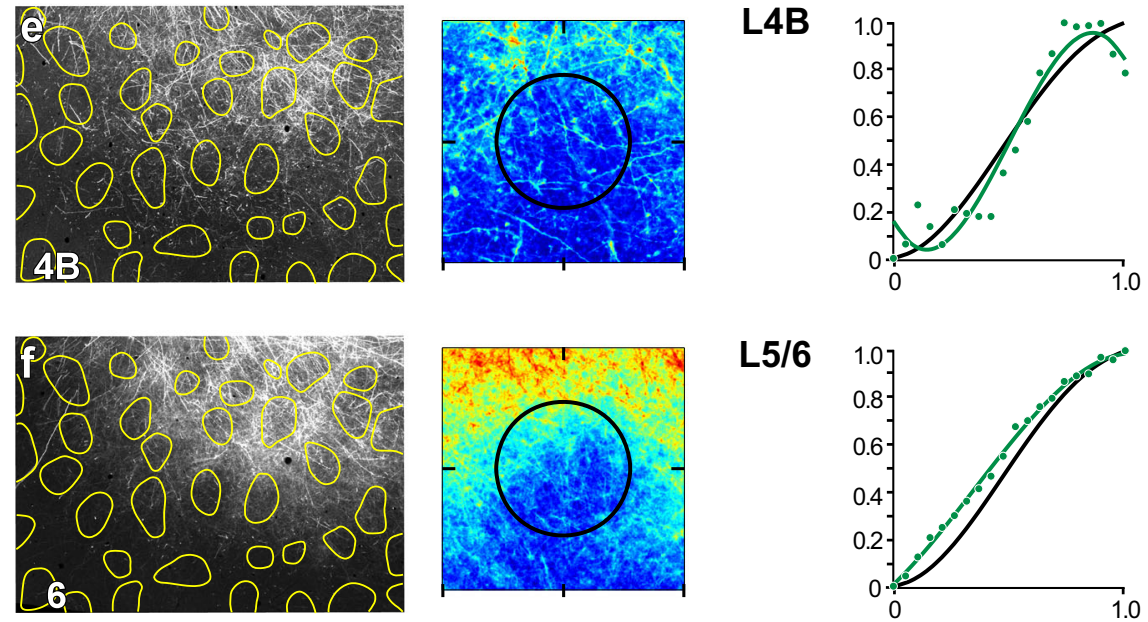

Fig. 6 V2 pale stripes project to V1 interblobs. Case MK374RHLat. Same as in Fig. 4, but for tdT-labeled FB axons in V1 resulting from the AAV9-tdT injection site confined to a pale-medial stripe shown in Fig. 2a-c. In all layers, GFP-label intensity dominates in the interblobs. Results in (a-f) are representative of three independent injections centered in pale stripes. Source data for right panels in (a-f) are provided as a Source data file. 


\section{a Thick Stripes}

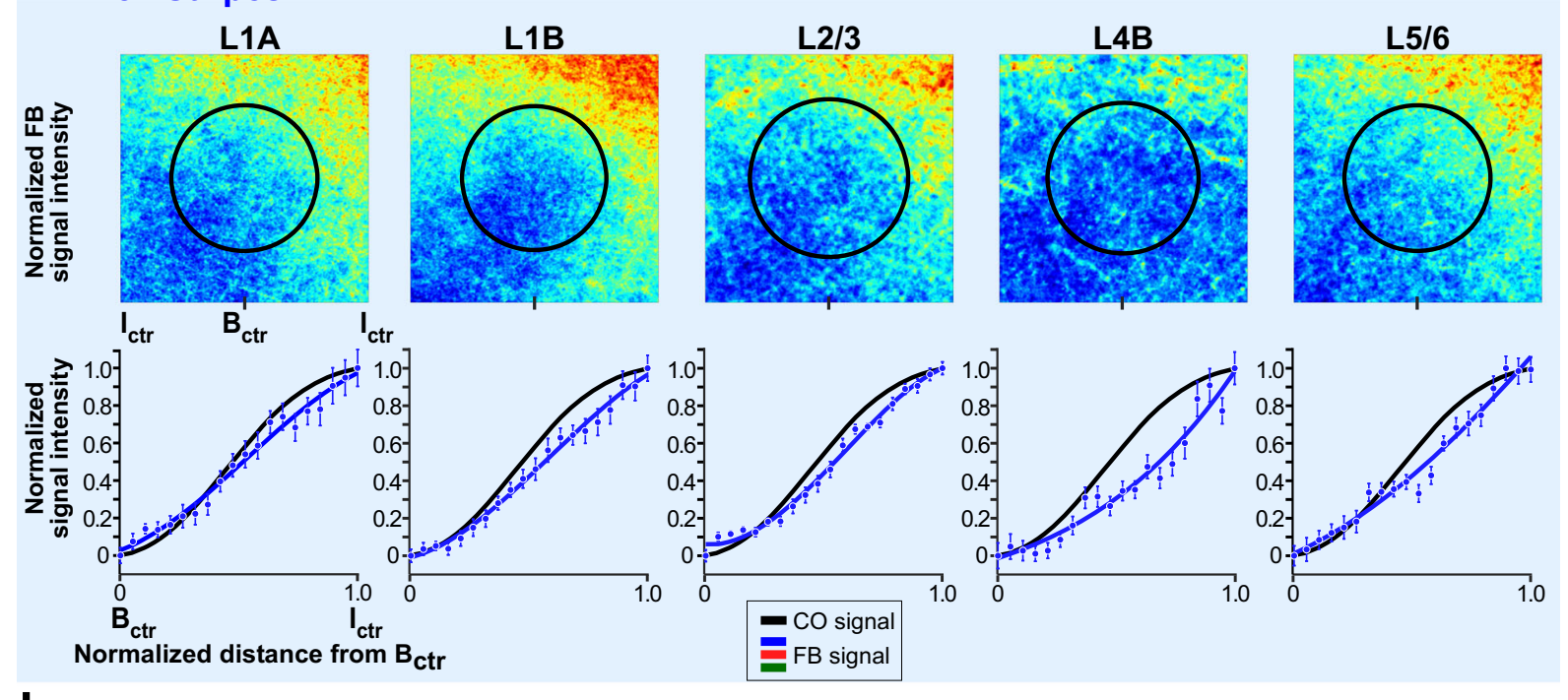

\section{b Thin Stripes}
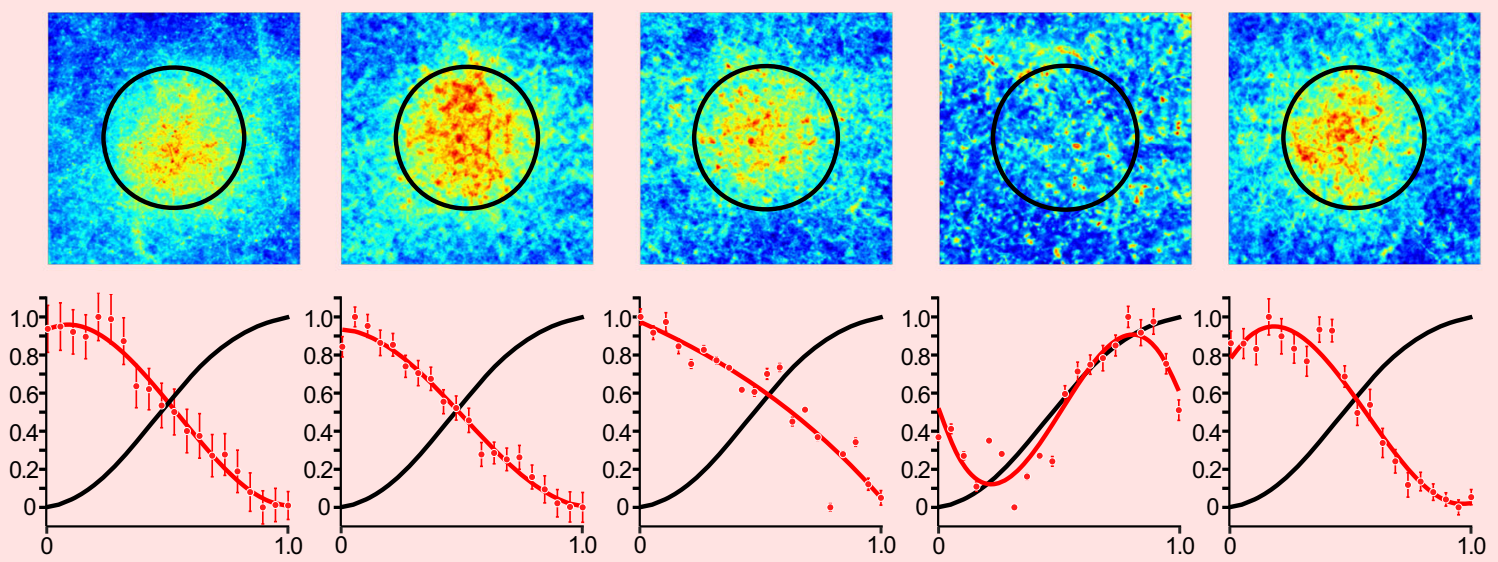

\section{Pale Stripes}
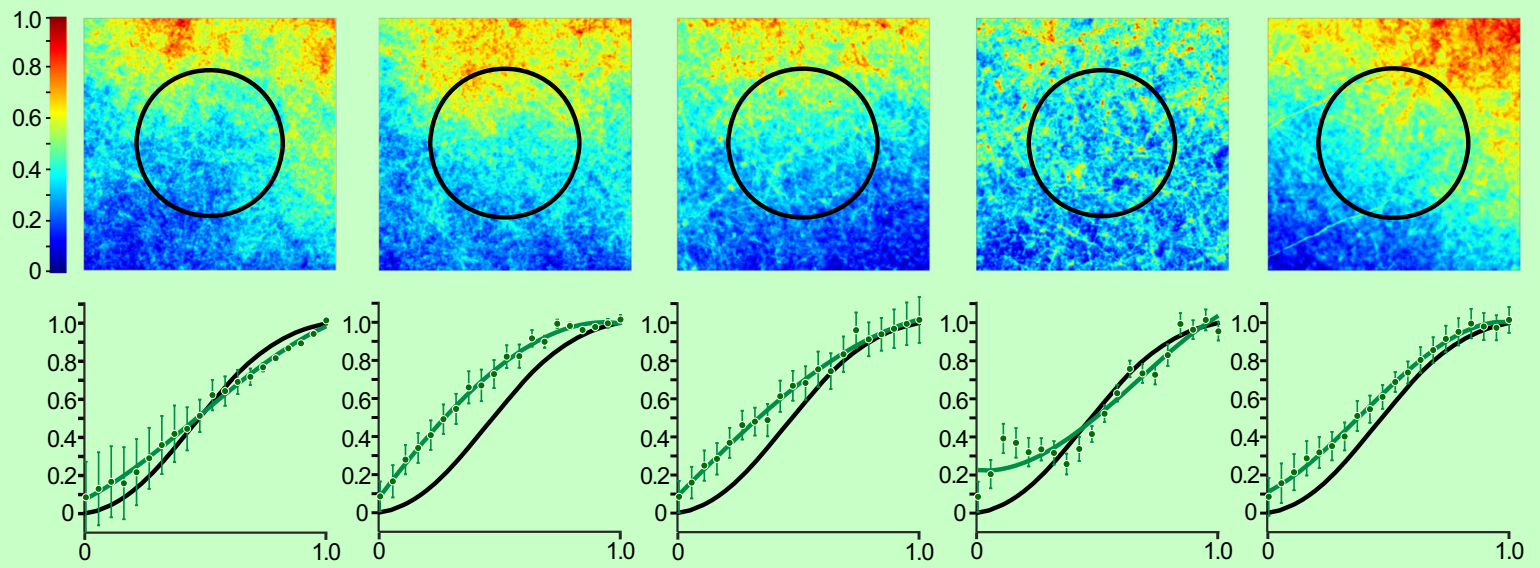

Fig. 7 Population analysis. Mean heatmaps of fluorescent signal intensity (top panels), and plots of fluorescent and CO signal intensities (bottom panels) for the population of FB projections arising from $\mathbf{a}$ V2 thick stripes $(n=3), \mathbf{b}$ thin stripes $(n=3)$, and $\mathbf{c}$ pale stripes $(n=3)$. For details of how the population means were computed see "Methods". Error bars in the fluorescent signal intensity plots are s.e.m. Statistical analysis of differences between stripe types are reported in the "Results". Conventions are as in Fig. 4. Source data for bottom panels in (a-c) are provided as a Source data file. 


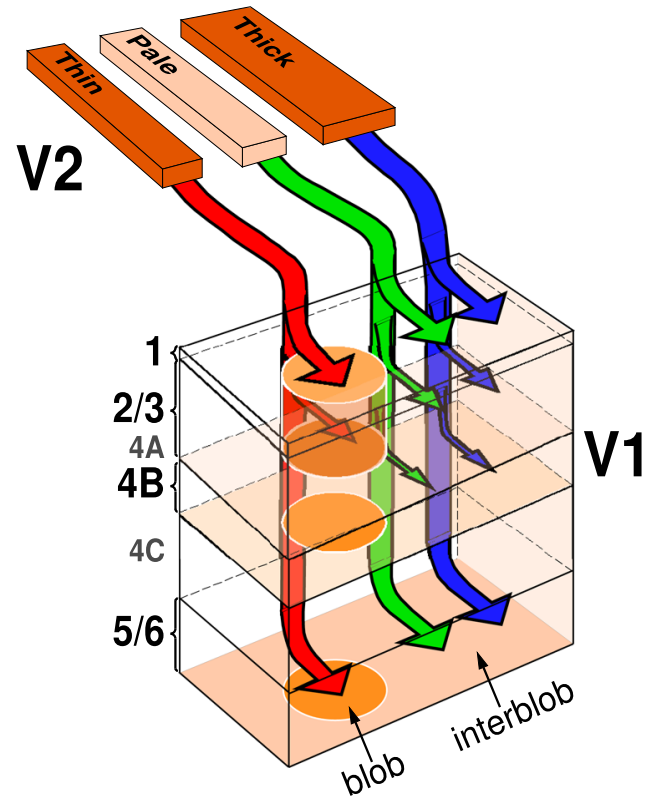

Fig. 8 Parallel V2-to-V1 feedback pathways. Our proposed model of the $\mathrm{FB}$ pathways from $\mathrm{V} 2$ to $\mathrm{V} 1$, based on the present study. The arrow thickness indicates the relative density of projections to the different $\mathrm{V} 1$ layers ( $L 4 A$ is omitted). For all stripe types, layers 1/upper 2 and 5/6 receive the densest $\mathrm{FB}$ projections, while lower $\mathrm{L} 2$ and $\mathrm{L} 3$ receive sparser projections from $\mathrm{V} 2$. Both thick (blue arrows) and pale (green arrows) stripes, but not thin (red arrows) stripes send FB projections to V1 L4B. FB projections from thin stripes predominantly target the $\mathrm{V} 1 \mathrm{CO}$ blob columns, while $\mathrm{FB}$ projections from thick and pale stripes predominantly target the $\mathrm{V} 1$ interblob columns.

predominantly CO-compartment specific, in agreement with Shmuel et al. ${ }^{27}$. These results are also consistent with previous demonstrations of patchy and CO-compartment-specific FB connections from area V3 to V1 in macaque ${ }^{11}$. In particular, we demonstrate that in macaque monkey, FB connections arising from the different $\mathrm{CO}$ stripes of V2 form parallel channels that mimic the reciprocal parallel pathways from V1 to V2 in this species $^{21}$. We also show that, with the exception of $\mathrm{L} 1$, the layers that give rise to $\mathrm{FF}$ connections to $\mathrm{V} 2(2 / 3,4 \mathrm{~A}, 4 \mathrm{~B}, 5 / 6)$ receive reciprocal $\mathrm{FB}$ inputs. This suggests that $\mathrm{V} 2 \mathrm{FB}$ axons may make direct contacts with V1 neurons sending FF inputs to V2, a hypothesis that we have recently confirmed using rabies virusmediated monosynaptic input tracing to label monosynaptic inputs to V2-projecting neurons in $\mathrm{V}^{36}$. However, while L4B has been shown to send at least some sparse inputs to thin and palemedial stripes $23,24,37$, we only observed significant FB inputs to L4B from thick and pale, but not thin, stripes. It is possible that our method of analysis failed to capture very sparsely labeled FB axons in L4B arising from thin stripes. Moreover, our low sample of injections into pale-medial stripes $(n=1)$ did not allow us to assess potential quantitative differences in the $\mathrm{FB}$ projections to L4B from the two pale-stripe types, as has been previously demonstrated for the L4B to pale-stripe FF projections ${ }^{23}$. In this study, we have not analyzed L4A for potential FB terminations, as this very thin layer is difficult to study in tangential sections, and in pia-to-white matter sections FB projections to L4A appeared to be, at best, very sparse.

Although here we are proposing the existence of at least three segregated FB pathways from V2 to V1, our study shows that FB projections from thick and pale stripes both terminate in the interblobs, allowing for the possibility that these projections contact the same V1 cells, therefore integrating information across these two parallel streams. However, current studies from our laboratory, using rabies virus-mediated monosynaptic input tracing to label direct inputs to V2-projecting neurons in V1, suggest that FB projections from thick and pale stripes contact predominantly distinct $\mathrm{V} 1$ projection neurons ${ }^{38}$.

Segregation of V1-to-V2 FF pathways within CO compartments likely reflects specialized function, because $\mathrm{CO}$ compartments in V1 and V2 show specialized neuronal responses properties and functional maps. Specifically, the blob-thin-stripe pathway is thought to process surface properties (color and brightness), the interblob-pale-stripe pathway object contours and form, and the interblob-thick stripe pathway object motion and depth ${ }^{31,39-50}$. Our finding of segregated FB projections within these same $\mathrm{CO}$ compartments, therefore, suggests that $\mathrm{V} 2 \mathrm{FB}$ connections to $\mathrm{V} 1$ do not integrate information across parallel functional streams, and thus across stimulus attributes, but rather modulate V1 responses to the specific stimulus attributes that are processed within their functional stream, an organization that may support, for example, feature-selective attention $^{51,52}$, or the feature specificity of extraclassical receptive field effects ${ }^{7,53,54}$.

Our finding of segregated V2-to-V1 FB pathways is consistent with previous results of stream-specific organization of corticogeniculate pathways in macaque ${ }^{55,56}$, suggesting this FB organization may exist at multiple levels of the visual pathway. Our results are also consistent with results in mouse visual cortex, demonstrating segregation of functional FB channels from L5 of higher visual areas AL (anterolateral) and PM (posteromedial) to $\mathrm{V}^{57}$, which also matches the functional segregation of the reciprocal FF pathways from V1 to AL and $\mathrm{PM}^{58}$. Modular FB axon terminations have also been demonstrated anatomically in L1 of mouse $\mathrm{V} 1$, where $\mathrm{FB}$ axons from areas $\mathrm{AL}$ and $\mathrm{PM}$ form interleaved terminal patches ${ }^{59}$. The FB patches from AL and PM, in turn align with M2 muscarinic acetylcholine receptor-rich and poor patches, respectively, which correspond to V1 regions specialized for the processing of distinct spatio-temporal features ${ }^{59,60}$. Interestingly, M2-positive patches are also found in macaque V1 L1 where they are in register with the CO-poor interblobs ${ }^{60}$. Together with our results, these studies support the notion of functionally specialized parallel FB pathways.

Our results of segregated $\mathrm{FB}$ pathways are consistent with predictive coding theories of FB function. According to these theories, the brain generates an internal model of the world, based on sensory data and prior experience, which is refined by incoming sensory data. The latter is compared to the predicted sensory data, and the prediction error ascends up the cortical hierarchy and refines the higher levels' model ${ }^{5,61,62}$. Predictive coding models postulate the existence of different neuronal populations encoding expectations and prediction errors, respectively, at each level of the cortical hierarchy. In these models, inter-areal FB connections carry the predictions, while FF connections carry the error in those predictions ${ }^{12,13}$. In terms of the architecture of hierarchical predictive coding schemes, one key attribute is the functional segregation of conditionally independent expectations, so that descending predictions are limited to prediction errors reporting a particular stimulus attribute, but not prediction errors reporting opposite or different attributes. For example, predictions about the orientation of a visual edge should not project to prediction errors units encoding visual motion, because knowing the orientation of an edge does not, statistically, tell us anything about its motion. This suggests that direct descending predictions will be largely specific for stimulus attributes (e.g., contours vs. motion), which is consistent with our finding of FB-specific channels. 
While our results strongly support the existence of functionally specialized parallel FB pathways, it is noteworthy that our method of analysis was designed to extract the densest FB terminations in V1. However, lighter FB axonal label was visible in the regions between the densest labeled terminal patches. Therefore, we cannot exclude that a smaller population of $\mathrm{FB}$ axons shows a different terminal pattern, such as projections to all $\mathrm{CO}$ compartments of V1 or projections to the opposite channel. This population of $\mathrm{FB}$ axons may serve to integrate information across streams, or to carry FB information about the same stimulus feature to neurons in different V1 CO compartments representing that feature, for example orientation in the blobs and interblobs. Similarly, FB from multiple stripes could affect the same neurons within the same stimulus feature, for example both thick and pale stripes have orientation maps, and FB to V1 from these stripes may be combined on the same V1 cells on the basis of orientation preference.

\section{Methods}

Experimental design. AAV9 vectors carrying the gene for either GFP or tdT were injected into specific V2 stripes, which in 3 animals were identified in vivo by intrinsic signal optical imaging. In two animals, instead, injections were made blindly with respect to stripe type. The V1 laminar and CO-compartment distribution of FB axons labeled by these viral injections was analyzed quantitatively.

Animals. Five adult (aged 2-5 yrs old) cynomolgus macaque monkeys (Macaca fasciculari; 4 females, 1 male) were used in this study. All procedures involving animals were approved by the Institutional animal Care and Use Committees of the University of Utah and conformed to the guidelines set forth by the USDA and NIH.

Surgical procedures. Animals were pre-anesthetized with ketamine (10-20 mg/kg, i.m.), intubated with an endotracheal tube, placed in a stereotaxic apparatus, and artificially ventilated. Anesthesia was maintained with isofluorane $(0.5-2.5 \%)$ in $100 \%$ oxygen, and end-tidal $\mathrm{CO}_{2}$, blood oxygenation level, electrocardiogram, and body temperature were monitored continuously. The scalp was incised, a craniotomy was made to expose the lunate sulcus and about $5 \mathrm{~mm}$ of cortex posterior to it (encompassing V2 and a small portion of V1). In two animals (MK359, 4 injections, and MK365, 3 injections) small durotomies were made just caudal to the posterior edge of the lunate sulcus, over V2, and viral vectors were injected into V2. In the reminder of cases ( 3 animals, total of 12 injections), optical imaging was used to identify the V2 stripes prior to the injections. In the latter cases, a large craniotomy and durotomy (15-20 $\mathrm{mm}$ mediolaterally, 6-8 $\mathrm{mm}$ anteroposteriorly) were performed to expose V2 and parts of V1, a clear sterile silicone artificial dura was placed on the cortex, and the craniotomy was filled with a sterile $3 \%$ agar solution and sealed with a glass coverslip glued to the skull with Glutures (Abbott Laboratories, Lake Bluff, IL). On completion of surgery, isoflurane was turned off and anesthesia was maintained with sufentanil citrate $(5-10 \mu \mathrm{g} / \mathrm{kg} / \mathrm{h}$, i.v.). The pupils were dilated with a short-acting topical mydriatic agent (tropicamide), the corneas protected with gas-permeable contact lenses, the eyes refracted, and optical imaging was started. Once the V2 stripes were functionally identified (1-3 h of imaging, see below), the glass coverslip, agar, and artificial dura were removed and the viral vectors were injected in specific V2 stripe types, using surface blood vessels as guidance. On completion of the injections, new artificial dura was placed on the cortex, the craniotomy was filled with Gelfoam and sealed with sterile parafilm and dental cement, the skin was sutured and the animal was recovered from anesthesia. Animals survived 3-4 weeks post-injections, and underwent a terminal 3-5 day optical imaging experiment to obtain additional functional maps. At the conclusions of this experiment the animal was sacrificed with Beuthanasia $(0.22 \mathrm{ml} / \mathrm{kg}$, i.v.) and perfused transcardially with saline for 2-3 min, followed by $4 \%$ paraformaldehyde in $0.1 \mathrm{M}$ phosphate buffer for $20 \mathrm{~min}$. One animal was perfused with $2 \%$ paraformaldehyde for $6 \mathrm{~min}$ and the brain postfixed overnight.

Injections of viral vectors. A total of 19 viral vector injections were made in 5 macaque monkeys. We excluded from analysis 6 injections, which either did not produce detectable label in V1 $(n=2)$, or were located in an unidentifiable stripe $(n=4)$. The viral vectors consisted of a 1:1 mixture of AAV9.CaMKIIa.4.Cre.SV40 and either AAV9.CAG.Flex.eGFP.WPRE.bGH or AAV9.CAG.Flex.tdTomato. WPRE.bGH (Penn Vector Core, University of Pennsylvania, PA), which were mixed and loaded in the same glass micropipette (tip diameter 35-45 $\mu \mathrm{m}$ ), and pressure injected using a picospritzer. For the injection cases used to investigate the distribution of FB terminals across V1 layers (Fig. $1 ; n=4$ ), we slowly injected (6-15 $\mathrm{nl}$ per $\mathrm{min}) 105-150 \mathrm{nl}$ of the viral mixture at a cortical depth of $1.2 \mathrm{~mm}$ from the pial surface. After a $5 \mathrm{~min}$ pause, the pipette was retracted to a depth of $0.5 \mathrm{~mm}$, and an additional $105-150 \mathrm{nl}$ of the viral mixture was injected at the same speed. The pipette was left in place for an additional 5-10 min before being retracted. These injection parameters yielded injection sites $1.3-1.8 \mathrm{~mm}$ in diameter encompassing all cortical layers. For the cases used to investigate the distribution of FB terminals relative to the V1 CO compartments, we used a similar procedure, but we injected smaller volumes in order to keep the injection site confined to a V2 stripe: $15-30 \mathrm{nl}$ were injected at $1.2 \mathrm{~mm}$ depth, and an additional $15-30 \mathrm{nl}$ at $0.5-0.6 \mathrm{~mm}$ depth. Resulting injection sites ranged in diameter between 0.56 and $0.95 \mathrm{~mm}$ and encompassed all V2 layers. One case used for the COcompartment analysis (MK356; Fig. 3) received a single injection of $105 \mathrm{nl}$ in a thin stripe and one injection of $75 \mathrm{nl}$ in a thick stripe, both at a cortical depth of $1 \mathrm{~mm}$ The resulting injection sites measured 1.26 and $0.65 \mathrm{~mm}$ in diameter, respectively, and encompassed all layers.

Each animal received 2-5 injections in dorsal area V2 of one hemisphere. Within the same hemisphere either vectors expressing different fluorophores (GFP or tdT) were injected, or injections of the same vector were spaced at least $10 \mathrm{~mm}$ apart, to ensure no overlap of the resulting labeled fields in V1. In one case (MK356), instead, two injections of the GFP-expressing vector were made in a thick and a thin stripe within the same V2 stripe cycle (Fig. 3), which resulted in two labeled fields in V1 that slightly overlapped (see Supplementary Fig. 2). In this case, we excluded from analysis the region of label overlap.

Optical Imaging. Acquisition of intrinsic signals was performed using the Imager 3001 and VDAQ software (Optical Imaging Ltd, Israel) under red light illumination $(630 \mathrm{~nm})$. To identify the V2 stripes, we imaged V2 during presentation of gratings varying in orientation and SF. Orientation maps were obtained by presenting for $4 \mathrm{~s}$ full-field, high contrast (100\%), pseudorandomized, achromatic drifting square-wave gratings of eight different orientations at 1.0-2.0 cycles $/^{\circ} \mathrm{SF}$, moving back and forth at 1.5 or 2 cycles/s in directions perpendicular to the grating orientation. Responses to same orientations were averaged across trials, following baseline correction, and difference images were obtained by subtracting the responses to two orthogonally oriented pairs (e.g., Figs. 2a, 3a). Imaging for SF (e.g., Figs. $2 \mathrm{a}$ and $3 \mathrm{a}$ ) was performed by presenting high contrast achromatic drifting square-wave gratings of 6 different SFs $\left(0.25,0.5,1,1.5,2,3\right.$ cycles $\left.^{\circ}{ }^{\circ}\right)$ alternating through 8 different orientations every $500 \mathrm{~ms}$, and drifting at 2 cycles/s. To create $\mathrm{SF}$ difference maps, baseline-corrected responses to high SF $\left(3 \mathrm{cycles} /{ }^{\circ}\right)$ were subtracted from baseline-corrected responses to low SF $\left(0.25\right.$ cycles $\left./{ }^{\circ}\right)$. Baseline correction for both the orientation and SF maps was performed in three different ways and the approach that provided the best maps was selected for analysis: (1) the baseline (pre-stimulus) was subtracted from the single condition response (i.e. the images recorded during stimulation of one stimulus orientation or one SF); (2) the single condition response was divided by the baseline; (3) the single condition response was divided by the "cocktail blank" (i.e., the average of responses to all oriented stimuli or all SF stimuli) 63,64 . Thick stripes were identified as the middle of regions having an orientation-preference map and domains responsive to low SFs; pale stripes as regions having an orientation-preference map neighboring a region with weak or no systematic orientation maps; and thin stripes as regions containing domains responsive to low SF, having weak or no systematic orientation maps (Figs. 2a, 3a). In each case, reference images of the surface vasculature were taken under green light $(546 \mathrm{~nm})$ illumination, and used in vivo as reference to position pipettes for viral vector injections, as well as postmortem to align the functionally identified V2 stripes to the histological sections containing the injection sites and sections labeled for CO to reveal the stripes (e.g., Figs. 2c, 3c, and Supplementary Fig. 1).

Histology. Areas V1 and V2 were dissected away from the rest of the visual cortex. The block was postfixed for $1-2 \mathrm{~h}$, sunk in $30 \%$ sucrose for cryoprotection, and frozen-sectioned at $40 \mu \mathrm{m}$. For the cases used to investigate the distribution of FB terminals across V1 layers (the cases in Fig. $1 ; n=4$ ), the block was cut perpendicularly to the layers and parallel to the V1-V2 border. This sectioning plane allows to easily identify layers, as well as the sequence of V2 stripes, which run perpendicular to the border. For the reminder of the cases $(n=9)$, which were used for the analysis of FB terminals relative to the V1 CO compartments, instead, the block was postfixed between glass slides for 1-2 $\mathrm{h}$ to slightly flatten the cortex in the optically imaged area, cryoprotected, and frozen-sectioned tangentially, paralle to the plane of the imaging camera. Sections were wet-mounted and imaged for fluorescent GFP and tdT-labeled cell bodies in V2 (the injection sites) and FB axons in $\mathrm{V} 1$, using either Zeiss Axioskop 2 or Axio Imager Z2 microscopes $(\times 10$ magnification; software: Zen -blue edition-, Carl Zeiss Microscopy GmbH). After digitizing the label, in most cases, the same sections were reacted for CO to reveal the cortical laminae and V1 and V2 compartments, and then re-imaged under bright field illumination $(\times 1.25-5$ magnification). In three cases, instead, the location of labeled axons for $2-3$ sections was determined by alignment to immediately adjacent CO-stained sections, rather than from the same section imaged for fluorescent label. In four cases, 1-2 sections were further immunoreacted to enhance the GFP signal strength, and then imaged a third time using both bright field and fluorescent channels to maintain a perfect alignment between the $\mathrm{CO}$ and label images. GFP immunohistochemistry was performed by incubating sections overnight at $4{ }^{\circ} \mathrm{C}$ in a chicken anti-GFP primary antibody (1:2000; RRID AB_10000240), followed by incubation in Alexa Fluor 488 Donkey Anti-Chicken IgG secondary antibody (1:200; RRID:AB_2340375). 


\section{Quantification and statistical analysis}

Analysis of injection site location. Digitized fluorescent label and CO staining from the same or adjacent sections were warped, using IR-tweak warping software (NCR toolset, Scientific Computing and Imaging Institute, University of Utah) by registering the radial blood vessels sequentially in a stack encompassing all cortical layers (for the tangentially sectioned cases). IR-tweak is an interactive, multithreaded, application for manual slice-to-slice registration. As control points are placed by the user in one image, their locations in the other image are estimated by the current thin-plate spline transform parameters ${ }^{65}$. When the user corrects the locations of estimated points in the second image, the transform parameters are updated. CO staining was used to assign injection sites to specific V2 CO stripes. To determine the CO stripe location of the injection sites, typically 3-6 images of serial CO sections were overlaid by aligning the radial blood vessels and merged in Adobe Photoshop. This approach reveals a more complete stripe pattern than any individual CO-stained section, in which the stripes can be incomplete. V2 injection sites were outlined through multiple images of serial injection sites, spanning the depth of the cortex; a composite injection site encompassing all outlines in individual sections was overlaid onto the merged $\mathrm{CO}$ stack, and its size and stripe location were determined. The size of the injections was defined as the region at the injection site where labeled cell bodies could be discerned, as the viral vectors we used infect neuronal somata. Dark CO stripes were classified as thick (or thin) if they appeared thicker (or thinner) than the two adjacent dark stripes in CO staining and coincided with thick (or thin) stripes as defined in the optical imaging maps (see above Optical Imaging), for cases in which imaging was available. To align CO-stained tissue and fluorescent images of the injection sites to the optical maps, we warped (using IR-tweak software) the most superficial tangential tissue sections containing the surface vessels (which run parallel to the cortical surface) to the image of the cortical surface vasculature obtained under green light (the latter in register with the functional maps obtained under red light). Each subsequent section was then aligned sequentially to the top sections by aligning the radial blood vessels (which run perpendicular to the cortical surface). This alignment procedure is illustrated in Supplementary Fig. 1, and was used to generate the images in Figs. $2 \mathrm{a}-\mathrm{c}$ and 3.

Laminar distribution of FB axons: quantitative analysis. For each injection case used for the laminar analysis $(n=4)$, the V1 laminar distribution of labeled V2 FB axons was analyzed in 3 tissue sections (cut perpendicular to the cortical layers), of which one section was selected near the center of the FB labeled field, where FB terminations are densest, and 1 section was selected on each side of this center section, equally spaced across the full extent of the labeled field in L4B (as the FB labeled field in this layer had a smaller tangential extent than the labeled fields in other layers, and we wanted the ROI chosen for analysis to include all layers of FB termination). Sections were imaged for GFP and tdT fluorescent label, stained for $\mathrm{CO}$ (to reveal layers), then re-imaged for both $\mathrm{CO}$ staining and fluorescent label. The layer boundaries were traced manually using $\mathrm{CO}$ staining. For each section selected for quantitative analysis, a ROI $(500 \mu \mathrm{m}$ wide, the full length of the cortex) was centered on the area that contained the densest fluorescent label. The ROI was further binned from top of the cortex to the white matter border into 100 bins. Individual pixel fluorescent intensity values were measured within each bin, and averaged to obtain the label intensity per bin. The different bin intensities were then normalized to the maximum bin intensity in that ROI The binning of the ROI provided a FB-label intensity profile across the cortical depth. The normalized label intensity in each bin was finally averaged across the 3 section ROIs and, again, normalized to the max average intensity to produce a single V1 laminar profile of the label intensity for each injection case. Population laminar profiles for FB arising from the same V2 stripe type were obtained by averaging the laminar profiles across cases with injections in the same stripe type. The cortical layer boundaries derived from the average layer thickness across COstained sections were then superimposed to the label laminar profile (right panels in Fig. 1e, f).

To determine whether viral injections in different V2 stripe types produced different laminar profiles, we performed statistical comparisons on a layer-by-layer basis. Because layers can vary in thickness across different sections, and therefore ROIs, in this analysis the same layer could contain slightly different numbers of bins across ROIs, each having an average pixel intensity value. The $n$ for a given layer dataset consisted of the average intensity value of each bin in that layer across all ROIs, pooled across all cases with an injection in the same V2 stripe type. For across stripe statistical comparison of FB-label intensity for each layer, we used a nonparametric test for two independent samples (Mann-Whitney U test-using the Matlab's ranksum function), due to the varying number of bins per layer and the non-normal distribution of the data.

For each stripe group, we also statistically compared the resulting FB-label intensity across the sublayers of $\mathrm{L} 4(4 \mathrm{~A}, 4 \mathrm{~B}, 4 \mathrm{C})$. The $n$ for the sublayer dataset consisted of the average intensity value of each bin in that sublayer across all ROIs, pooled across all cases with an injection in the same V2 stripe. For statistical comparison we used the Kruskal-Wallis test with a Bonferroni corrected $\alpha$ value of 0.017 .

CO-compartment distribution of FB axons: quantitative analysis. This analysis was performed on tissue sectioned tangentially to the pial surface. For each injection case used for this analysis ( $n=9$ injections, 3 for each stripe type), digitized images of fluorescent label and CO staining were warped and aligned into a sequential stack through the depth of the cortex, as described above for the analysis of V2 injection site location. CO staining was used to identify layers, as well as the blobs and interblobs in the superficial layer sections. For each case, we selected for quantitative analysis at least one section (the one with the densest label) through each of the following layers: 1A, 1B, 2/3, 4B, 5/6. However, for some layers (particularly the thinner L1A, $1 \mathrm{~B}$, and $4 \mathrm{~B}$ ), in some cases it was necessary to select for analysis two adjacent sections, because the cut was not perfectly tangential and therefore the label field in that layer was split between adjacent sections; this ensured that the entire labeled FB field for that layer was analyzed. To quantify the distribution of labeled FB axons across V1 CO compartments within each of the chosen sections, we extracted from a L2/3 CO-stained section in the aligned section stack the $\mathrm{CO}$ blob borders, blob centers and interblob center-lines (Supplementary Fig. 3a). Blob borders were extracted by processing digitized images of CO-stained sections using low-pass and bell-shaped local histogram equalization filters (using Image Pro Plus Inc. software, Media Cybernetics Inc.); the latter filter gets rid of inequalities in CO-staining intensity across the tissue by distributing the image histogram evenly around the center of the histogram intensity scale. This image manipulation results in a more uniform image intensity distribution across the imaged region (e.g., Supplementary Fig. 2e), from which one can, then, segment out the blobs as the darkest $33 \%$ of pixels across the gray scale image (yellow

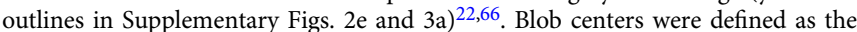
darkest spot within each blob (red dots in Supplementary Fig. 3a), as determined by the image analysis software; the latter were then visually inspected for accuracy and manually corrected if necessary. For blobs that showed significant elongation, we marked several centers; the latter generally coincided with multiple peaks in CO intensity along the length of the blob, having similar center-to-center spacing as the surrounding tissue (e.g., see several elongated blobs in Supplementary Fig. 3a). The centers of the interblobs were defined as the brightest pixels between adjacent blob centers (green outlines in Supplementary Fig. 3a).

For each injection case, we analyzed the CO map in V1 L2/3 encompassing the full tangential extent of the labeled FB fields across the layers. From this CO map we estimated a square region of interest (ROI; black box in Supplementary Fig. 3a) whose size encompassed the average blob and interblob size in the map. To estimate this ROI, for each blob in the CO map, we computed a mean blob centerto-interblob center $\left(\mathrm{B}_{\mathrm{ctr}}-\mathrm{I}_{\mathrm{ctr}}\right)$ distance from radial distances measured at $20^{\circ}$ increments around the blob center. A grand average $B_{c t r}-I_{c t r}$ distance was then computed from all relevant blobs' mean $\mathrm{B}_{\mathrm{ctr}}-\mathrm{I}_{\mathrm{ctr}}$ distances. This grand average distance was doubled to obtain the ROI for that case. This ROI was then centered on each blob in the CO map, and the CO-staining intensity was measured for each pixel within each blob ROI, summed across all blob ROIs, and normalized to the maximum summed intensity value, to produce a $\mathrm{CO}$-intensity heatmap for the case (Supplementary Fig. 3b). Mean blob diameter for the case was computed from all blobs used for the analysis and overlaid onto the CO heatmap (black circle in Supplementary Fig. 3b).

In a similar fashion we generated heatmaps of fluorescent FB-label (GFP or tdT) intensity for each layer of FB termination, by measuring fluorescent intensity within the same size ROI used to compute the CO heatmap, centered on each blob in the CO map that overlaid the labeled FB field in that layer (Supplementary Fig. 3c). Therefore, the number of blobs used for this analysis could vary from layer to layer, depending on the extent and location of the labeled fields in each layer. Signal intensity was summed across all ROIs in the layer, and normalized to the maximum intensity, to produce a fluorescent intensity heatmap for that layer (Supplementary Fig. 3d). A mean blob diameter specific to each layer was computed from all blobs used to generate each layer heatmap, and overlaid onto the layer heatmap (black circle in Supplementary Fig. 3d).

From the heatmaps, we generated graphs of the normalized $\mathrm{CO}$ or fluorescence signal intensity as function of distance from the blob center (Supplementary Fig. 3e). Because the distribution of FB label was not homogenous all around the blobs (e.g., Supplementary Fig. 3d), these graphs were generated by measuring the average signal intensity in each of 20 bins spanning from the center of the blob (zero on the $x$-axis) to the center of the interblob (1.0 on the $x$-axis) within a 200 $\mu \mathrm{m}$-wide window centered in the region of highest signal intensity in the heatmap (black boxes in Supplementary Fig. 3b, d); signal intensity was finally normalized to the maximum bin intensity value. The region of highest intensity on the heatmap was determined by measuring the average signal intensity within a $200-\mu \mathrm{m}$-wide window rotated every $20^{\circ}$ around the blob center, and selecting the window with the highest average signal intensity. As the latter was measured independently on the $\mathrm{CO}$ and fluorescence heatmaps, their respective windows of highest signal intensity did not necessarily match in location around the average blob center (e.g., in Supplementary Fig. $3 \mathrm{~d}$, the window is located at $90^{\circ}$, while in Supplementary Fig. 3b it is located at $290^{\circ}$ ).

To produce population heatmaps for each cortical layer (Fig. 7), the layer heatmaps from each case were scaled up to the largest heatmap across the relevant cases, summed and normalized to the maximum summed value. The population graphs in Fig. 7 were generated from the population heatmaps as described above for the individual cases (Supplementary Fig. 3e).

We statistically compared the location of densest FB label produced by injections in different stripe type. To this purpose, for each case layer heatmap, within the $200 \mu \mathrm{m}$ window of densest label, out of the 20 bins we selected for 
analysis those with $>70 \%$ intensity. The locations of the selected bins relative to the blob center were compared layer by layer across stripe types. For statistical comparison across stripes we used a Kruskal-Wallis test with a Bonferroni corrected $\alpha$ value of 0.017 . We also performed a statistical comparison by pooling all the selected bin locations across all layers for a given stripe type and comparing across stripe types, again using Kruskal-Wallis test with Bonferroni correction.

Reporting summary. Further information on research design is available in the Nature Research Reporting Summary linked to this article.

\section{Data availability}

The data that support the findings of this study are available from the corresponding author upon reasonable request. Source data are provided with this paper.

\section{Code availability}

The Matlab code used to generate the heatmaps and perform data analyses in this study are deposited on GitHub ${ }^{67}$.

Received: 10 March 2020; Accepted: 3 December 2020;

Published online: 11 January 2021

\section{References}

1. Van Essen, D. C. \& Maunsell, J. H. R. Hierarchical organization and functional streams in the visual cortex. Trends Neurosci. 6, 370-375 (1983).

2. Hubel, D. H. \& Wiesel, T. N. Receptive fields, binocular interaction and functional architecture in the cat's visual cortex. J. Physiol. 160, 106-154 (1962).

3. McAdams, C. J. \& Reid, C. R. Attention modulates the responses of simple cells in monkey primary visual cortex. J. Neurosci. 25, 11023-11033 (2005)

4. Luck, S. J., Chelazzi, L., Hillyard, S. A. \& Desimone, R. Neural mechanims of spatial selective attention in areas V1, V2 and V4 of macaque visual cortex. J. Neurophysiol. 77, 24-42 (1997).

5. Rao, R. P. \& Ballard, D. H. Predictive coding in the visual cortex: a functional interpretation of some extra-classical receptive-field effects. Nat. Neurosci. 2, 79-87 (1999).

6. Friston, K. A theory of cortical responses. Philos. Trans. R. Soc. Lond. B Biol. Sci. 360, 815-836 (2005).

7. Angelucci, A. et al. Circuits and mechanisms for surround modulation in visual cortex. Ann. Rev. Neurosci. 40, 425-451 (2017).

8. Nurminen, L., Merlin, S., Bijanzadeh, M., Federer, F. \& Angelucci, A. Topdown feedback controls spatial summation and response amplitude in primate visual cortex. Nat. Commun. 9, 2281 (2018).

9. Hupé, J. M. et al. Cortical feedback improves discrimination between figure and background by V1, V2 and V3 neurons. Nature 394, 784-787 (1998).

10. Nassi, J. J., Lomber, S. G. \& Born, R. T. Corticocortical feedback contributes to surround suppression in V1 of the alert primate. J. Neurosci. 33, 8504-8517 (2013).

11. Angelucci, A. \& Bressloff, P. C. The contribution of feedforward, lateral and feedback connections to the classical receptive field center and extra-classical receptive field surround of primate V1 neurons. Prog. Brain Res. 154, 93-121 (2006).

12. Bastos, A. M. et al. Canonical microcircuits for predictive coding. Neuron 76, 695-711 (2012).

13. Shipp, S. Neural elements for predictive coding. Front. Psychol. 7, 1792 (2016).

14. Mumford, D. On the computational architecture of the neocortex. II. The role of cortico-cortical loops. Biol. Cyber. 66, 241-251 (1992).

15. Roelfsema, P. R. Cortical algorithms for perceptual grouping. Ann. Rev. Neurosci. 29, 203-227 (2006).

16. Hawkins, J. On Intelligence (Times Books, 2004).

17. Livingstone, M. S. \& Hubel, D. H. Anatomy and physiology of a color system in the primate visual cortex. J. Neurosci. 4, 309-356 (1984)

18. Livingstone, M. S. \& Hubel, D. H. Connections between layer $4 \mathrm{~B}$ of area 17 and the thick cytochrome oxidase stripes of area 18 in the squirrel monkey. J. Neurosci. 7, 3371-3377 (1987).

19. Sincich, L. C. \& Horton, J. C. The circuitry of V1 and V2: integration of color, form and motion. Ann. Rev. Neurosci. 28, 303-326 (2005).

20. Roe, A. W., Lu, H. D. \& Chen, G. in Encyclopedia of Neuroscience (ed. Squire, L.) (Elsevier, 2008).

21. Sincich, L. C. \& Horton, J. C. Divided by cytochrome oxidase: a map of the projections from V1 to V2 in macaques. Science 295, 1734-1737 (2002).

22. Federer, F. et al. Four projections streams from primate V1 to the cytochrome oxidase stripes of V2. J. Neurosci. 29, 15455-15471 (2009).
23. Federer, F., Williams, D., Ichida, J. M., Merlin, S. \& Angelucci, A. Two projection streams from macaque V1 to the pale cytochrome oxidase stripes of V2. J. Neurosci. 33, 11530-11539 (2013)

24. Sincich, L. C., Jocson, C. M. \& Horton, J. C. V1 interpatch projections to V2 thick stripes and pale stripes. J. Neurosci. 30, 6963-6974 (2010).

25. Angelucci, A. et al. Circuits for local and global signal integration in primary visual cortex. J. Neurosci. 22, 8633-8646 (2002).

26. Angelucci, A., Levitt, J. B. \& Lund, J. S. Anatomical origins of the classical receptive field and modulatory surround field of single neurons in macaque visual cortical area V1. Prog. Brain Res. 136, 373-388 (2002).

27. Shmuel, A. et al. Retinotopic axis specificity and selective clustering of feedback projections from V2 to V1 in the owl monkey. J. Neurosci. 25 2117-2131 (2005).

28. Stettler, D. D., Das, A., Bennett, J. \& Gilbert, C. D. Lateral connectivity and contextual interactions in macaque primary visual cortex. Neuron 36, 739-750 (2002).

29. Maunsell, J. H. R. \& Van Essen, D. C. The connections of the middle temporal visual area (MT) and their relationship to a cortical hierarchy in the macaque monkey. J. Neurosci. 3, 2563-2586 (1983).

30. Rockland, K. S. \& Pandya, D. N. Laminar origins and terminations of cortical connections of the occipital lobe in the Rhesus monkey. Brain Res. 179, 3-20 (1979).

31. Livingstone, M. S. \& Hubel, D. H. Segregation of form, color, movement, and depth: anatomy, physiology, and perception. Science 240, 740-749 (1988).

32. Rockland, K. S. \& Virga, A. Terminal arbors of individual "feedback" axons projecting from area V2 to V1 in the macaque monkey: a study using immunohistochemistry of anterogradely transported phaseolus vulgarisleucoagglutinin. J. Comp. Neurol. 285, 54-72 (1989).

33. Rockland, K. S. \& Knutson, T. Feedback connections from area MT of the squirrel monkey to areas V1 and V2. J. Comp. Neurol. 425, 345-368 (2000).

34. Anderson, J. C. \& Martin, K. A. C. The synaptic connections between cortical areas V1 and V2 in macaque monkey. J. Neurosci. 29, 11283-11293 (2009).

35. Luo, L., Callaway, E. M. \& Svoboda, K. Genetic dissection of neural circuits. Neuron 57, 634-660 (2008).

36. Siu, C., Balsor, J., Federer, F. \& Angelucci, A. A direct feedback-to-feedforward circuit in primate visual cortex. BioRxiv https://doi.org/10.1101/ 2020.07.07.192450 (2020).

37. Sincich, L. C., Jocson, C. M. \& Horton, J. C. Neurons in V1 patch columns project to V2 thin stripes. Cereb. Cortex 17, 935-941 (2007).

38. Siu, C., Merlin, S., Federer, F. \& Angelucci, A. A direct feedback-tofeedforward circuit for fast feedback modulation of incoming feedforward signals. SFN Abstracts online 219.22 (2018).

39. DeYoe, E. A. \& Van Essen, D. C. Segregation of efferent connections and receptive field properties in visual area V2 of the macaque. Nature 317, 58-61 (1985).

40. Ts'o, D. Y. \& Gilbert, C. D. The organization of chromatic and spatial interactions in the primate striate cortex. J. Neurosci. 8, 1712-1727 (1988)

41. Yoshioka, T. \& Dow, B. M. Color, orientation and cytochrome oxidase reactivity in areas V1, V2 and V4 of macaque monkey visual cortex. Behav. Brain Res. 76, 71-88 (1996).

42. Tootell, R. B., Silverman, M. S., Hamilton, S. L., De Valois, R. L. \& Switkes, E. Functional anatomy of macaque striate cortex. III. Color. J. Neurosci. 8 , 1569-1593 (1988).

43. Tootell, R. B., Nelissen, K., Vanduffel, W. \& Orban, G. A. Search for color "center(s)" in macaque visual cortex. Cereb. Cortex 14, 353-363 (2004).

44. Xiao, Y., Casti, A., Xiao, J. \& Kaplan, E. Hue maps in primate striate cortex. Neuroimage 35, 771-786 (2007).

45. Xiao, Y., Wang, Y. I. \& Felleman, D. J. A spatially organized representation of color in macaque cortical area V2. Nature 421, 535-439 (2003).

46. Wang, Y. I., Xiao, Y. \& Felleman, D. J. V2 thin stripes contain spatially organized representations of achromatic luminance change. Cereb. Cortex 17, 116-129 (2007).

47. Chen, G., Lu, H. D. \& Roe, A. W. A map for horizontal disparity in monkey V2. Neuron 58, 442-450 (2008).

48. Lu, H. D., Chen, G., Tanigawa, H. \& Roe, A. W. A motion direction map in macaque V2. Neuron 68, 1002-1013 (2010).

49. Lu, H. D. \& Roe, A. W. Functional organization of color domains in V1 and V2 of macaque monkey revealed by optical imaging. Cereb. Cortex 18, 516-533 (2008).

50. Landisman, C. E. \& Ts'o, D. Y. Color processing in macaque striate cortex: relationships to ocular dominance, cytochrome oxidase, and orientation. J. Neurophysiol. 87, 3126-3137 (2002).

51. Maunsell, J. H. \& Treue, S. Feature-based attention in visual cortex. Trends Neurosci. 29, 317-322 (2006).

52. Moore, T. \& Zirnsak, M. Neural Mechanisms of Selective Visual Attention. Annu. Rev. Psychol. 68, 47-72 (2017).

53. Angelucci, A. \& Shushruth, S. in The New Visual Neurosciences (eds Chalupa, L. M. \& Werner, J. S.) (MIT Press, 2013). 
54. Albright, T. D. \& Stoner, G. R. Contextual influences on visual processing. Ann. Rev. Neurosci. 25, 339-379 (2002).

55. Briggs, F., Kiley, C. W., Callaway, E. M. \& Usrey, W. M. Morphological substrates for parallel streams of corticogeniculate feedback originating in both V1 and V2 of the macaque monkey. Neuron 90, 1-12 (2016).

56. Briggs, F. \& Usrey, W. M. Parallel processing in the corticogeniculate pathway of the macaque monkey. Neuron 62, 135-146 (2009).

57. Huh, C. Y. L., Peach, J. P., Bennett, C., Vega, R. M. \& Hestrin, S. Featurespecific organization of feedback pathways in mouse visual cortex. Curr. Biol. 28, 114-120 (2018).

58. Glickfeld, L. L., Andermann, M. L., Bonin, V. \& Reid, R. C. Cortico-cortical projections in mouse visual cortex are functionally target specific. Nat. Neurosci. 16, 219-226 (2013).

59. D’Souza, R. D., Bista, P., Meier, A. M., Ji, W. \& Burkhalter, A. Spatial clustering of inhibition in mouse primary visual cortex. Neuron 104, 588-600 (2019).

60. Ji, W. et al. Modularity in the organization of mouse primary visual cortex. Neuron 87, 632-643 (2015).

61. Friston, K. Hierarchical models in the brain. PLoS Comput. Biol. 4, e1000211 (2008).

62. Friston, K. \& Kiebel, S. Predictive coding under the free-energy principle. Philos. Trans. R. Soc. Lond. B Biol. Sci. 364, 1211-1221 (2009).

63. Bonhoeffer, T. \& Grinvald, A. in Brain Mapping: the Methods (eds Toga, A. W. \& Mazziotta, J. C.) (Academic Press, 1996).

64. Shmuel, A. \& Grinvald, A. Functional organization for direction of motion and its relationship to orientation maps in cat area 18. J. Neurosci. 16, 6945-6964 (1996).

65. Anderson, J. R. et al. A computational framework for ultrastructural mapping of neural circuitry. PLoS Biol. 7, 493-512 (2009).

66. Boyd, J. D. \& Casagrande, V. A. Relationships between cytochrome oxidase (CO) blobs in primate primary visual cortex (V1) and the distribution of neurons projecting to the middle temporal area (MT). J. Comp. Neurol. 409, 573-591 (1999).

67. Federer, F., Ta'afua, S., Merlin, S., Hassanpour, M. \& Angelucci, A. Streamspecific feedback inputs to the primate primary visual cortex matlab code. https://doi.org/10.5281/zenodo.4267029 (2020).

\section{Acknowledgements}

We thank Kesi Sainsbury for expert technical assistance, and Dr. Jeff Yarch for help with some experiments. We also thank Drs. Stewart Shipp and Karl J. Friston (UCL) for helpful discussions on predictive coding and the role of feedback. Supported by grants from NIH (R01 EY026812, R01 EY019743, BRAIN U01 NS099702), NSF (IOS 1755431, EAGER 1649923), University of Utah Research Foundation and Neuroscience Initiative to A.A., and from Research to Prevent Blindness, Inc. and a core grant from NIH (EY014800) to the Department of Ophthalmology, University of Utah.

\section{Author contributions}

Conceptualization: all authors. Methodology: all authors. Software: S.T. and M.S.H Validation: S.T., F.F., and A.A. Formal Analysis: S.T. and M.S.H. Investigation: F.F., S.M., and A.A. Writing—original draft: F.F., S.T., and A.A. Writing-review/editing: all authors. Visualization: F.F. and S.T. Supervision \& funding acquisition: A.A.

\section{Competing interests}

The authors declare no competing interests.

\section{Additional information}

Supplementary information is available for this paper at https://doi.org/10.1038/s41467 020-20505-5.

Correspondence and requests for materials should be addressed to A.A.

Peer review information Nature Communications thanks Amir Shmuel and the other, anonymous reviewer(s) for their contribution to the peer review of this work.

Reprints and permission information is available at http://www.nature.com/reprints

Publisher's note Springer Nature remains neutral with regard to jurisdictional claims in published maps and institutional affiliations.

Open Access This article is licensed under a Creative Commons Attribution 4.0 International License, which permits use, sharing, adaptation, distribution and reproduction in any medium or format, as long as you give appropriate credit to the original author(s) and the source, provide a link to the Creative Commons license, and indicate if changes were made. The images or other third party material in this article are included in the article's Creative Commons license, unless indicated otherwise in a credit line to the material. If material is not included in the article's Creative Commons license and your intended use is not permitted by statutory regulation or exceeds the permitted use, you will need to obtain permission directly from the copyright holder. To view a copy of this license, visit http://creativecommons.org/ licenses/by/4.0/.

(C) The Author(s) 2021 